\title{
Analytical and Computational Studies of Intramolecular Electron Transfer Pertinent to Electron Transfer and Electron Capture Dissociation Mass Spectrometry ${ }^{\dagger}$
}

\author{
Diane Neff and Jack Simons* \\ Chemistry Department and Henry Eyring Center for Theoretical Chemistry University of Utah, \\ Salt Lake City, Utah 84112
}

Received: June 17, 2009; Revised Manuscript Received: August 7, 2009

\begin{abstract}
Earlier work from this group has suggested that, in electron capture and electron-transfer mass spectrometry experiments on positively charged gas-phase samples of polypeptides, the initial electron attachment event most likely occurs at one of the peptide's positively charged sites (e.g., protonated side chains), although electron attachment can occur at a disulfide or amide site ca. 1-10\% of the time. Focusing on the 90-99\% dominant channel in which initial electron attachment occurs at a positive site, this paper addresses to what extent and over what distances electron transfer can take place from a positively charged site to a disulfide $\sigma^{*}$ or amide $\pi^{*}$ orbital, because it is thought that it is through such orbitals that disulfide or $\mathrm{N}-\mathrm{C}_{\alpha}$ backbone bond cleavage occurs. Ab initio electronic structure calculations show that, as long as an SS $\sigma^{*}$ (or OCN $\pi^{*}$ ) orbital experiences sufficient Coulomb stabilization from proximal positively charged groups, there are a myriad of excited Rydberg states located on positive sites that are able to induce such intrapeptide electron transfer. Computational data show that the transfer rates decay exponentially with distance for a given Rydberg orbital. An analytical model is developed that allows us to estimate the rates of Rydberg-to-valence and Rydberg-to-Rydberg electron transfers as functions of the Rydberg orbitals' $n$ quantum numbers. This model suggests that transfer can occur over very long distances at rates that are more than competitive with the rates of radiationless relaxation within the manifold of Rydberg states (the latter processes eventually terminate the electron-transfer process an thus the disulfide or $\mathrm{N}-\mathrm{C}_{\alpha}$ bond cleavages), and it gives formulas for how these rates depend on $n$ (and thus the radial span of the Rydberg orbitals).
\end{abstract}

\section{Introduction and Background}

This research group has been involved since the early $1970 \mathrm{~s}^{1}$ in the study of negative molecular ions and electron-molecule interactions, and in 2008 produced a review article ${ }^{2}$ overviewing much of the theoretical and experimental research that has taken place in the study of such species in recent years. This history of work on molecular anions makes our participation in this special issue devoted to Professor Carl Lineberger especially timely and exciting to us. The present work deals with yet another adventure in this field: how electrons (either free or from an anion donor) can induce very specific bond cleavages when attached to a molecule containing one or more positively charged groups. In particular, the work presented here relates to two very new forms of mass spectrometry that show great promise for sequencing peptides, which, of course, is an important contributor to the field of proteomics.

Specifically, electron-capture dissociation ${ }^{3}$ (ECD) and electrontransfer dissociation ${ }^{4}$ (ETD) mass spectroscopic methods have shown much utility and promise for sequencing peptides and proteins. A strongpoint of both techniques is their propensity for selectively cleaving disulfide and $\mathrm{N}-\mathrm{C}_{\alpha}$ bonds and for doing so over a wide range of the backbone, thus producing many different fragment ions, unlike collision-induced dissociation (CID) or infrared multiphoton dissociation (IRMPD) where fewer distinct fragment ions are usually formed. ECD and ETD also preserve labile side chains with post-translational modifications, which is another positive attribute. Parallel with many

\footnotetext{
† Part of the "W. Carl Lineberger Festschrift".

* Corresponding author. E-mail: simons@chem.utah.edu; http://simons. hec.utah.edu.
}

advances in the experimental development and improvement of these methods, theoretical studies have been carried out by several groups in several nations to try to determine the mechanism $(\mathrm{s})^{5}$ by which electron attachment leads to these specific bond cleavages as well as how the initial electron attachment occurs.

Key questions that have been the focus of some of these theoretical studies are

1. At what sites in the gas-phase multiply charged polypeptide can the ECD or ETD electron attach? Are some sites favored over others?

2. After electron attachment to one site occurs, can the electron subsequently migrate to other site(s)? If so, over what distance is migration feasible?

3. What is the molecular-level mechanism by which the attached electron induces the disulfide or $\mathrm{N}-\mathrm{C}_{\alpha}$ bond cleavage observed in ECD/ETD experiments?

Before describing the contributions from the present work, for the remainder of this Introduction, we explain the Coulomb stabilization model that has formed the basis of much of this group's contributions to the field. We offer this explanation to illustrate how this model was used in our earlier work to address the three questions posed above and because it again plays a central role in the present work. Then, in section II, we describe the strategy we employ in making use of model compounds to address these and other questions, and we explain why we are forced to limit our calculations to model compounds rather than full polypeptides. In section III, we summarize the most important findings that have been obtained in earlier studies, and we discuss how the present work adds to this body of 
SCHEME 1: Direct Electron Attachment to a Coulomb Stabilized S-S $\sigma^{*}$ or OCN $\pi^{*}$ Orbital To Cleave a Disulfide or $\mathrm{N}-\mathrm{C}_{\alpha}$ Bond
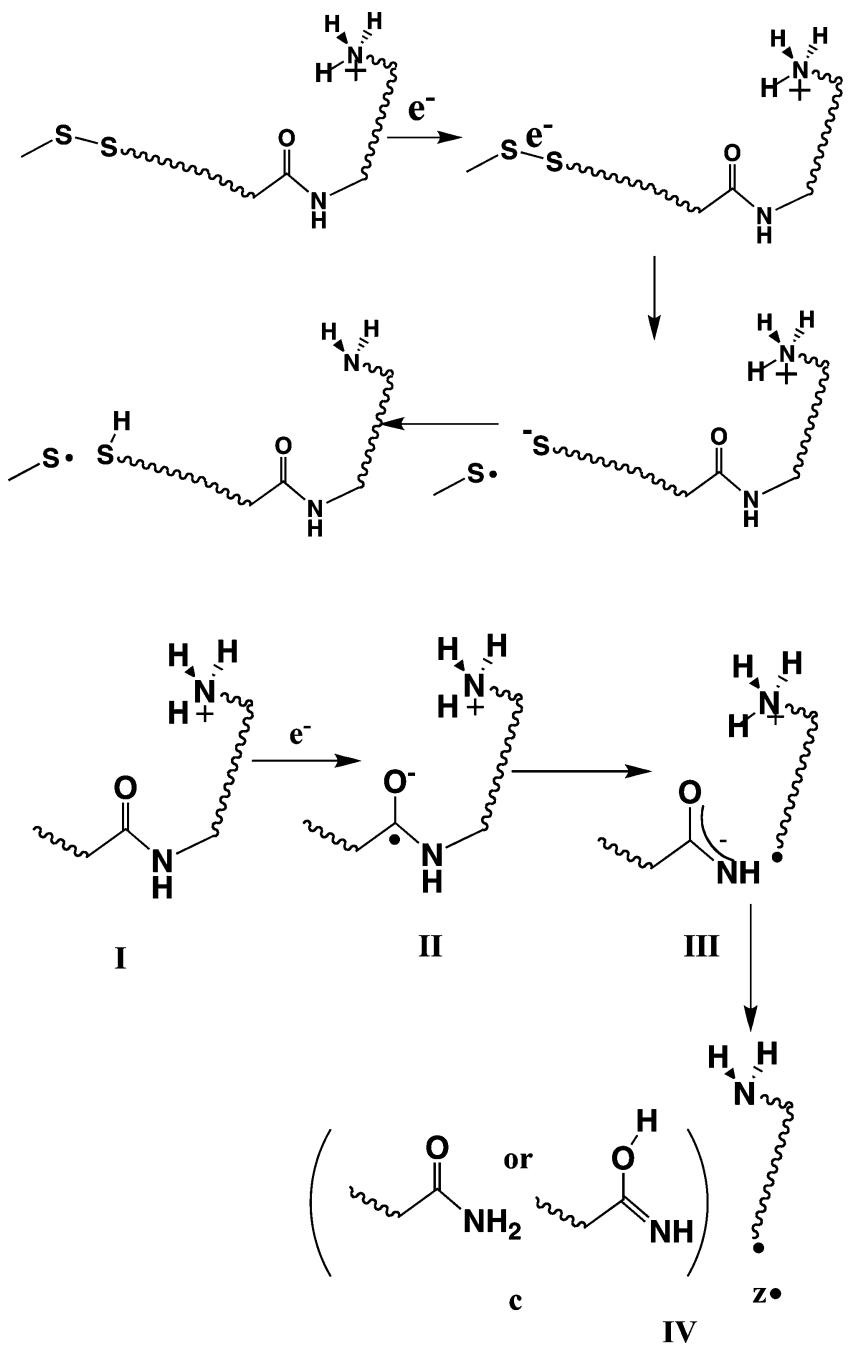

evidence. Section IV describes the theoretical methods used in the present study. In section $\mathrm{V}$ we present the computational and analytical results of the present study, and, in section VI, we summarize how our present findings contribute to "the big picture" of how ETD and ECD function.

A. Coulomb Stabilization Postulate. At an early stage of our efforts ${ }^{5 \mathrm{~h}-\mathrm{m}, \mathrm{o}-\mathrm{t}}$ to explore how ETD or ECD electrons can cleave $\mathrm{S}-\mathrm{S}$ or $\mathrm{N}-\mathrm{C}_{\alpha}$ bonds in positively charged gas-phase peptides, we proposed that electrons can attach directly (i.e., in a nearly vertical exothermic process) to $\mathrm{S}-\mathrm{S} \sigma^{*}$ or $\mathrm{OCN}$ amide $\pi^{*}$ orbitals, but only under special conditions. In particular, we suggested that such low-lying antibonding orbitals can have their energies lowered ${ }^{6}$ by attractive Coulomb interactions with positively charged groups (e.g., the protonated amine or fixedcharge groups on side chains) thus rendering the electron attachment exothermic. We first put forth this model in 2003 to explain SS cleavage, $;^{5 \mathrm{j}}$ later, $\mathrm{we}^{5 \mathrm{~h}-\mathrm{m}, \mathrm{O}-\mathrm{t}}$ and the Turecek group $^{5 \mathrm{a}-\mathrm{f}}$ extended the model to electron attachment to amide $\pi^{*}$ orbitals relating to $\mathrm{N}-\mathrm{C}_{\alpha}$ bond cleavage. In Scheme 1, we illustrate the mechanisms by which such direct electron attachment events have been proposed to lead to cleavage of disulfide or $\mathrm{N}-\mathrm{C}_{\alpha}$ bonds through what we termed Coulomb-stabilized direct electron attachment and what is sometimes called the UW (Utah-Washington) mechanism.
In the $\mathrm{N}-\mathrm{C}_{\alpha}$ cleavage case, two possible structures for the c-type fragments are shown; they have identical mass-to-charge ratios, so the mass spectrometry experiment cannot distinguish them. The $\mathrm{O}=\mathrm{C}-\mathrm{NH}_{2}$ amide structure (left) is thermodynamically more stable, but depending whether a proton is transferred from elsewhere in the polypeptide before or after the $\mathrm{N}-\mathrm{C}_{\alpha}$ bond cleaves, one would expect either the enol-imine (right) or the amide (left) to be formed, respectively. For example, if the proton transfer occurs prior to $\mathrm{N}-\mathrm{C}_{\alpha}$ cleavage, the negative oxygen center in the structure labeled II in Scheme 1 would be expected to be protonated and thus the enol-imine would be formed. Recently, an infrared multiphoton action spectroscopic probe $^{7}$ of the c-type fragments formed in ECD was carried out and it was determined that the amide structure is formed, not the enol-imine. However, the workers of ref 7 point out that it is possible the amide structure could be formed by isomerization of the initially formed enol-imine species (i.e., there is enough energy available to effect this isomerization). So, this spectroscopic data provide evidence suggesting (but not proving) that the proton transfer takes place after the $\mathrm{N}-\mathrm{C}_{\alpha}$ bond has cleaved, not before.

B. Origins of the Coulomb Postulate. What caused us to suggest the Coulomb stabilization model was others' work on dissociative electron attachment ${ }^{8}$ showing that, in the absence of Coulomb stabilization, vertical electron attachment to an $\mathrm{S}-\mathrm{S}$ $\sigma^{*}$ or amide $\pi^{*}$ orbital is ca. $1 \mathrm{eV}$ and ca. $2.5 \mathrm{eV}$ endothermic, respectively. So, we knew that very low-energy free electrons (as in ECD where most of the electrons are thought to have kinetic energy far below $1 \mathrm{eV}$ ) would not be likely to vertically attach; this, of course, suggests it would be even less feasible for ETD electrons to attach (because the electron binding energy of the anion donor must also be surmounted). Knowing that the Coulomb potential varies with distance $R$ as $14.4 \mathrm{eV} \AA / R$ with $R$ in $\AA$, we postulated that a disulfide linkage must experience Coulomb stabilization exceeding $1 \mathrm{eV}$ for ECD (even more for ETD) to render our direct-attachment mechanism feasible; this stabilization could arise, for example, from a single positively charged site closer than ca. $14 \AA$, from two positive sites each $28 \AA$ distant, or from a doubly charged site $28 \AA$ away. In a multiply charged polypeptide, all of the Coulomb potentials contribute to this stabilization. Analogously, we postulated that a single positive charge 14.4/2.5 $=6 \AA$ from an OCN $\pi^{*}$ orbital could render this orbital amenable to exothermic direct ECD electron attachment.

As we now explain, the Coulomb potentials generated by all of the positively charged groups in an ETD or ECD polypeptide influence both (i) the energy landscapes pertinent to the electronic states arising in the collision of an ETD donor anion (or an ECD free electron) with a positively charged polypeptide and (ii) the energy landscapes relating to intrapeptide electron transfer from Rydberg states localized on a positive site to SS $\sigma^{*}$ or OCN $\pi^{*}$ orbital sites.

C. Coulomb Stabilization's Influence on Intrapeptide Electron Transfer. One of the effects of Coulomb stabilization is illustrated in Figure 1 where we show energy profiles (as functions of the SS bond length) for states of a model peptide system in which

a. no electron has yet attached (this is called the parent system),

b. an electron has attached to the SS $\sigma^{*}$ orbital, or

c. an electron has attached to a Rydberg orbital (ground or excited) on the protonated Lys side chain.

As stated above, the SS $\sigma^{*}$-attached state's curve is expected to lie ca. $1 \mathrm{eV}$ above the energy of the parent near the 


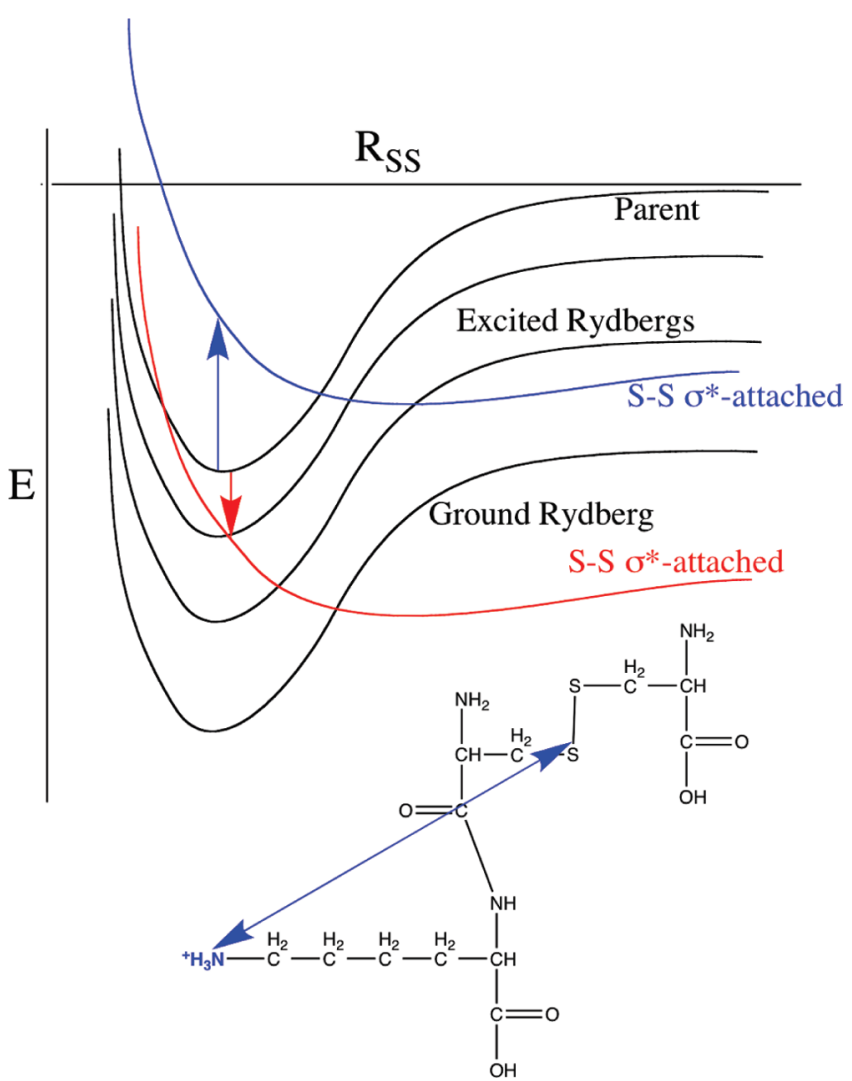

Figure 1. Qualitative depictions, as functions of the SS bond length, of the energies of the model peptide shown above with no electron attached (labeled parent), with an electron attached to a ground or excited Rydberg orbital (three curves parallel to that of the parent but shifted to lower energies), or with an electron attached to the SS $\sigma^{*}$ orbital (blue and red) (appears as Figure 1 in ref $5 \mathrm{~s}$, reprinted with permission, copyright 2008, Elsevier).

equilibrium SS bond length, as shown by the blue curve in Figure 1. However, the Coulomb potential exerted by the protonated Lys site shifts the SS $\sigma^{*}$-attached curve downward in energy to produce the red curve shown in Figure 1. Of course, such a peptide in the gas phase will undergo very large geometry changes caused by its internal thermal energy, so the distance $R$ from the Lys' nitrogen atom to the SS bond will undergo corresponding dynamical changes. These intramolecular dynamical motions will cause the Coulomb stabilization of the SS $\sigma^{*}$-attached state to also fluctuate; at some times, the red curve in Figure 1 may lie vertically (i.e., at the parent's equilibrium geometry) above the energy of the parent, while at other times, it can lie below the parent.

As we discuss in more detail later, in each of our studies on model compounds, ${ }^{5 \mathrm{~h}-\mathrm{m}, \mathrm{o}-\mathrm{t}}$ we carry out calculations in which the total Coulomb stabilization at an SS (or OCN) bond site is held fixed (to represent an instantaneous geometrical arrangement of a polypeptide's charges). The locations of these charges are chosen to generate a range of Coulomb potentials that would be experienced within a real polypeptide undergoing thermal motions. For each such charge locations, we then attempt to determine

a. whether direct electron attachment to the SS $\sigma^{*}$ (or OCN $\pi^{*}$ ) orbital is energetically feasible (i.e., not endothermic) and, if so, what the probability (and cross-section) for such a direct attachment is, and

b. which Rydberg states are intersected by the SS $\sigma^{*-}$ (or OCN $\pi^{*}$-) attached state near the equilibrium geometry of the parent polypeptide. This tells us which Rydberg states to
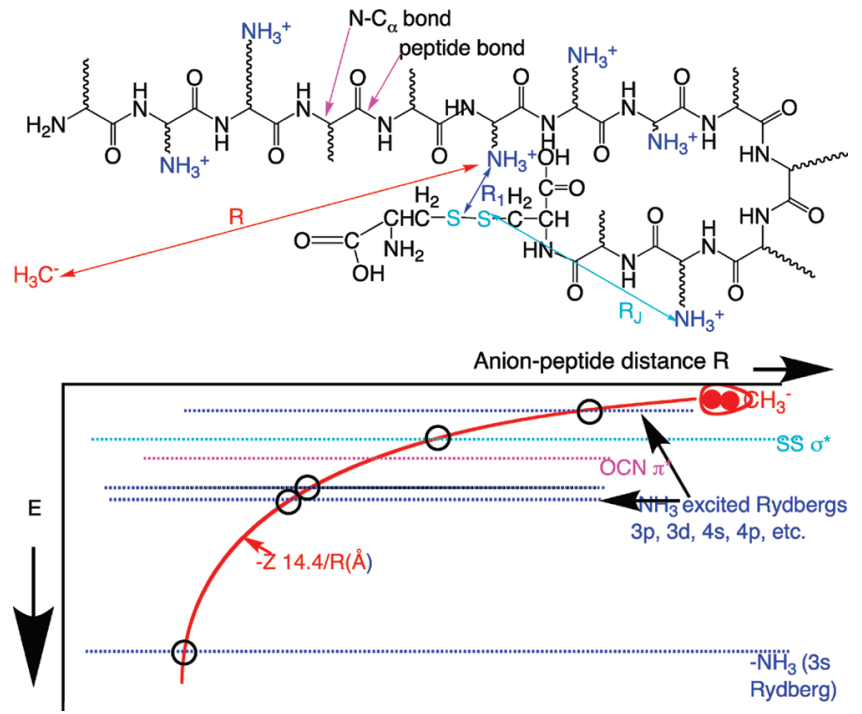

Figure 2. Qualitative depiction of the energies (as functions of the anion-peptide separation $R$ ) of the ion-pair state (red), states in which an electron has transferred from the anion donor to a Rydberg (ground $3 \mathrm{~s}$ or excited 3p, 3d, 4s, 4p, etc.) orbital, and states in which the electron has transferred from the donor anion to an OCN $\pi^{*}$ or SS $\sigma^{*}$ orbital.

consider when we take into account that the electron may initially attach to a Rydberg orbital on a positive site and subsequently transfer to an SS or OCN site. Estimating these intrapeptide electron-transfer rates require us to locate the crossings of the SS $\sigma^{*}$ (or OCN $\pi^{*}$ ) surfaces with the vertically accessible Rydberg states and to evaluate the electronic coupling strengths $\left(H_{1,2}\right)$ between the crossing states as discussed later in this paper.

For the example shown in Figure 1, direct attachment is feasible because the red curve lies vertically below the parent, and it is the second excited Rydberg state, not the ground or first excited Rydberg state, that might provide the means for electron transfer from the protonated Lys to the SS bond to occur (i.e., because the ground and first excited Rydberg curves are intersected by the red curve at a geometry too far from the parent's equilibrium).

D. Coulomb Stabilization's Influence on the Initial Electron Attachment. The intrapeptide Coulomb potentials also play a key role in determining the energy landscapes pertinent to the ETD or ECD initial electron attachment process. In Figure 2 we show a model polypeptide containing both a disulfide linkage, several $\mathrm{N}-\mathrm{C}_{\alpha}$ bonds, and several positively charged groups, and we show a $\mathrm{H}_{3} \mathrm{C}^{-}$anion donor undergoing a collision with the multiply charged peptide.

In the bottom part of Figure 2, we show qualitative depictions of some of the electronic states that relate to the electron-transfer event as functions of the distance $R$ between the anion donor and the peptide having charge $Z$. The rapidly descending red curve shows how the energy of the ion-pair state corresponding to $\mathrm{H}_{3} \mathrm{C}^{-}$and the $Z$-charged peptide varies with $R$; it follows the attractive Coulomb form $-14.4 \mathrm{Z} \mathrm{eV/R}(\AA)$. The other curves shown in Figure 2 are meant to show the $R$ dependences of states in which an electron has been transferred from the $\mathrm{H}_{3} \mathrm{C}^{-}$ anion donor to (i) ground (3s) or (ii) excited (3p, 3d, $4 \mathrm{~s}, 4 \mathrm{p}$, etc.) Rydberg orbitals localized on one ${ }^{9}$ of the peptide's positive sites, (iii) the SS $\sigma^{*}$ orbital, or (iv) one of the peptide's OCN $\pi^{*}$ orbitals. All of these states' energy profiles are shown as being rather independent of $R$ (at least at large $R$ ) because they relate to interactions of a neutral $\mathrm{H}_{3} \mathrm{C}$ radical with a peptide 
having charge $(Z-1)$. Of course, at shorter $R$ values, valencerange interactions set in and cause these curves to eventually (at small $R$ ) become repulsive.

The large- $R$ energies of the OCN $\pi^{*}$ - and SS $\sigma^{*}$-attached states relative to that of the parent peptide of charge $Z$ are determined by the intrinsic energies of these two orbitals $(1 \mathrm{eV}$ for the former, $2.5 \mathrm{eV}$ for the latter) stabilized by the total Coulomb potential

$$
C=-14.4 \mathrm{eV} \sum_{J} \frac{Z_{J}}{R_{J}(\AA)}
$$

Here, $Z_{J}$ is the charge on the $J$ th charged site and $R_{J}$ is the distance (in $\AA$ ) from that $J$ th site to the SS or OCN site whose Coulomb stabilization one is evaluating. Likewise, the energy of each of the Rydberg-attached states will depend on the intrinsic electron binding energy for that state stabilized by the Coulomb potential at that Rydberg site due to all of the other charged sites in the polypeptide.

Clearly, as the polypeptide undergoes thermal intramolecular motions, these Coulomb potentials will vary and, as a result, the locations of the crossings between the ion-pair state's potential and the potentials of the SS $\sigma^{*}$-, OCN $\pi^{*}$, or Rydbergattached states will vary with time. As discussed earlier, we carry out calculations on a series of model compounds in each of which the locations of the positive sites and thus the total Coulomb potential are held fixed. It is by finding the crossing points mentioned above (for a series of model compounds) and using Landau-Zener theory ${ }^{5 \mathrm{~h}, \mathrm{i}, \mathrm{m}}$ to estimate the probabilities for surface hopping at these crossings that we have been able to estimate the rates for electron transfer from an anion donor such as $\mathrm{H}_{3} \mathrm{C}^{-}$to a Rydberg, $\mathrm{SS} \sigma^{*}$, or $\mathrm{OCN} \pi^{*}$ orbital. We will have more to say later about such calculations when we summarize the results of our findings to date.

\section{Computational Strategy of Our Studies}

As already briefly discussed, in all of the electronic structure simulations that we have carried out, we have not tried to simulate the full dynamical motions of the backbone and side chains of polypeptides as they undergo electron attachment, subsequent intrapeptide electron migration, and bond cleavage. We need to treat most if not all of the functional groups within the peptide using ab initio electronic structure rather than molecular mechanics methods because we have to allow the attaching electron to go wherever it "wants". Moreover, we have to employ flexible atomic orbital basis sets to allow for the large radial extent of the Rydberg and antibonding orbitals involved in the electron transfer. Thus, it is simply not computationally feasible for us to carry out fully electronic structure based studies of even small polypeptides in which the dynamical motions of all their atoms are included.

Instead, we have taken an approach in which we carry out calculations on series of model compounds in which

a. the functional group whose bond cleavage is being probed (e.g., $\mathrm{SS}$ or $\mathrm{N}-\mathrm{C}_{\alpha}$ ) is present,

b. one or more positively charged groups are also present (to generate the Coulomb stabilization and to provide a manifold of Rydberg orbitals that might attach and/or transfer an electron), and

c. within each model compound, the distance(s) from the positive site(s) to the SS or $\mathrm{N}-\mathrm{C}_{\alpha}$ bond are fixed.

For example, we show in Figure 3 data from a model study of SS bond cleavage in which we used model compounds

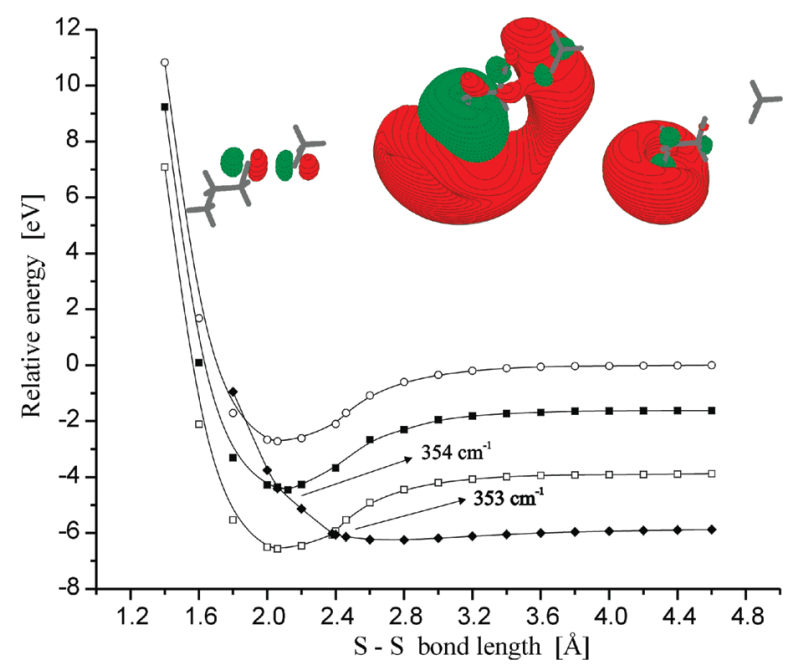

Figure 3. Energy profiles for parent (open circles), ground 3s-attached (open squares), excited 3p-attached (filled squares) Rydberg and SS $\sigma^{*}$-attached states of $\mathrm{H}_{3} \mathrm{C}-\mathrm{SS}-\left(\mathrm{CH}_{2}\right)_{n}-\mathrm{NH}_{3}{ }^{+}$for $n=2$. Also shown are the SS $\sigma^{*}$ (left), 3p Rydberg (center), and 3s Rydberg (right) orbitals as well as the $H_{1,2}$ coupling matrix elements connecting the SS $\sigma^{*}$ and other states at their avoided crossings (appears as Figure 3 in ref 5p, reproduced with permission; copyright 2008, Elsevier).

$\mathrm{H}_{3} \mathrm{C}-\mathrm{SS}-\left(\mathrm{CH}_{2}\right)_{n}-\mathrm{NH}_{3}{ }^{+}$having the protonated site separated from the SS bond by aliphatic linkages, among which the distance $R_{\mathrm{NS}}$ between the protonated amine and the nearest $\mathrm{S}$ atom varies (as the methylene chain length varies) but in which $R_{\mathrm{NS}}$ is held fixed (for a given compound) as the SS bond is elongated to generate the energy landscapes illustrated in Figure 3.

In such studies, we focused primarily on learning

a. which Rydberg state(s) cross the SS $\sigma^{*}$-attached state near the parent's equilibrium geometry because it is these Rydberg states that we found provide the most efficient avenue for transferring an electron from a positive site to an SS (or OCN) bond site, and

b. the magnitude of the $H_{1,2}$ electronic couplings ${ }^{10}$ between pairs of adiabatic states that undergo crossings near the parent's equilibrium geometry.

A. Electron-Transfer Rates as Functions of $\boldsymbol{H}_{1,2}$. For the example shown in Figure 3, it is the 3p excited Rydberg state that offers the best chance for coupling to the SS $\sigma^{*}$-attached state, and its $H_{1,2}$ coupling $\left(354 \mathrm{~cm}^{-1}\right)$ can be used to estimate (with Landau-Zener (LZ) theory) the rate of transfer of the attached electron from the $3 \mathrm{p}$ Rydberg to the SS $\sigma^{*}$ orbital. In LZ theory, the probability $\mathrm{P}$ for hopping from one electronic state to another at a point of avoided crossing is given as

$$
P=1-\exp \left[-\frac{2 \pi H_{1,2}^{2}}{\hbar v|\Delta F|}\right] \approx \frac{2 \pi H_{1,2}^{2}}{\hbar v|\Delta F|}
$$

where $H_{1,2}$ is one-half the splitting as the two energy surfaces undergo their avoided crossing, $v$ is the speed at which the coordinate $R$ moves through the avoided crossing region, and $\Delta F$ is the difference in the slopes of the two energy surfaces as they approach the avoided crossing. When eq 2 is applied to the intrapeptide electron-transfer events illustrated in Figure 3, we multiplied this probability expression by the frequency $v$ with which the SS bond vibrates through a crossing region ${ }^{11}$ to estimate the rate of electron transfer: 


$$
\begin{aligned}
\text { rate } & =1.8 \times 10^{13}\left\{1-\exp \left[-\frac{2 \pi H_{1,2}^{2}}{\hbar v|\Delta F|}\right]\right\} \mathrm{s}^{-1} \\
& \approx 1.8 \times 10^{13} \frac{2 \pi H_{1,2}^{2}}{\hbar v|\Delta F|} \mathrm{s}^{-1}
\end{aligned}
$$

with the approximation in the right of eq 3 pertaining when $P$ is much smaller than unity (as it is in all of the cases we have encountered). Taking $v$ as the average speed of motion of the SS bond in its lowest vibrational level, and calculating $\Delta F$ from the slope differences between the Rydberg and SS $\sigma^{*}$ curves at the avoided crossing, we found ${ }^{5 \mathrm{~s}}$ that these rates can be estimated by using the simplified formula

$$
\text { rate } \approx 10^{12}\left[\frac{H_{1,2}\left(\mathrm{~cm}^{-1}\right)}{300}\right]^{2} \mathrm{~s}^{-1}
$$

This simplified expression's validity derives from the facts that

(i) the slope differences $\Delta F$ for Rydberg states (all of which are nearly parallel to one another) intersecting the SS $\sigma^{*}$ state are essentially equal to the slope of the SS $\sigma^{*}$ state because the slope of the Rydberg state near the parent's equilibrium geometry is small, and

(ii) the slope of the $\mathrm{SS} \sigma^{*}$ state varies little as it crosses the various Rydberg states.

However, it is important to note that the scaling formula shown in eq 4 relates to the Rydberg-to-SS $\sigma^{*}$ electron-transfer rates involving only $3 \mathrm{~s}$ or $3 \mathrm{p}$ Rydberg orbitals separated (by distance $R_{\mathrm{NS}}$ ) from the SS bond by 1, 2, or 3 methylene spacer units. In the present paper, we will have more to say about how $H_{1,2}$ varies as the principal quantum number $n$ of the Rydberg orbital varies. Such knowledge is important because, for example, $3 \mathrm{~s}$ and $3 \mathrm{p}$ Rydberg orbitals may have sufficient radial extent to couple to an SS $\sigma^{*}$ orbital in one distance range, but it might require a larger Rydberg orbital (e.g., 4s, 4p, 4s, or 6s) to span larger distances.

B. $H_{1,2}$ Values Decay Exponentially with Distance. As noted earlier, we found that the $H_{1,2}$ couplings decay exponentially with the distance $R_{\mathrm{NS}}$ between the protonated amine site and the SS bond site, as expected. The exponential decay slopes are not the same among various Rydberg states, but they are of similar magnitude for $3 \mathrm{~s}$ and $3 \mathrm{p}$ Rydberg states, and are found to drop to $H_{1,2} \approx 0.3 \mathrm{~cm}^{-1}$ when $R_{\mathrm{NS}} \approx 15-20 \AA$ for both $3 \mathrm{~s}$ and 3 p states. According to eq $4, H_{1,2}$ values near $0.3 \mathrm{~cm}^{-1}$ represent the limit below which intrapeptide electron transfer (e.g., from a Rydberg orbital on a protonated amine to an SS $\sigma^{*}$ orbital) can compete with intrapeptide radiationless decay of excited Rydberg states (that take place at rates of ca. $10^{6}$ $\mathrm{s}^{-1}$ ) leading eventually to the ground 3s Rydberg state, which is known to dissociate in ca. $10^{-9}$ s (e.g., $\mathrm{R}-\mathrm{NH}_{3} \rightarrow \mathrm{H}+$ $\mathrm{R}-\mathrm{NH}_{2}$ or $\rightarrow \mathrm{R}+\mathrm{NH}_{3}$ ), thus terminating the possibility of further intrapeptide electron transfer. It is for this reason that $H_{1,2}$ must be larger than ca. $0.3 \mathrm{~cm}^{-1}$ if intrapeptide electron transfer can be expected to generate $\mathrm{SS}$ ( or $\mathrm{N}-\mathrm{C}_{\alpha}$ ) bond cleavage.

C. Important Earlier Findings Supporting the Coulomb Stabilization Model. The Coulomb-stabilized direct attachment model was used in our first effort (ref $5 \mathrm{j}$ ) to rationalize the distribution of fragment ions observed ${ }^{12}$ in the Marshall lab under ECD conditions for the doubly charged peptides shown in Figure 1. In this species, two positively charged sites (protonated or sodiated Lys) are held rather far (up to $30 \AA$ ) from a disulfide bond by rigid polyalanine helices.
The importance of the experimental findings in this case relates to an earlier proposal from the McLafferty lab $^{3 a}$ that is called the Cornell mechanism. In this mechanism, as applied to the species shown in Figure 4, an electron is captured at one of the two protonated Lys sites to form a Rydberg species that subsequently undergoes $\mathrm{H}$ atom loss from one of its $-\mathrm{NH}_{3}{ }^{+}$ termini. The ejected $\mathrm{H}$ atom then attacks either the $\mathrm{S}-\mathrm{S}$ bond to cleave it (generating an $-\mathrm{S}^{\bullet}$ radical and an $\mathrm{H}-\mathrm{S}-$ unit) or the oxygen of a nearby amide carbonyl group to generate a $-\left({ }^{\circ} \mathrm{COH}\right)-\mathrm{NH}-$ carbon radical. This carbon radical then undergoes $\mathrm{N}-\mathrm{C}_{\alpha}$ cleavage to generate the enol-imine c-type fragment shown in Scheme 1. The recent observation that c-type fragments are likely (albeit not definitely) formed as amides rather than enol-imines and the observation ${ }^{12}$ that substantial SS bond cleavage occurs for the species shown in Figure 1 even when 20 alanines are present (and the distance from the putative nascent $\mathrm{H}$ atom and the SS bond is ca. $30 \AA$ ) was used ${ }^{5 j}$ to argue against the Cornell mechanism and in favor of the UW mechanism. Finally, it should be noted that the main bond cleavages observed for such species were (i) SS bond cleavage (ca. $70-75 \%$ of the fragmentation events) and (ii) $\mathrm{N}-\mathrm{C}_{\alpha}$ cleavage but only in the four alanines closest to the terminal lysines; both of these cleavages are consistent with the Coulomb stabilization model requiring 1 and $2.5 \mathrm{eV}$ of stabilization, respectively.

\section{Summary of Results from Earlier Studies}

The most important findings from our earlier studies of model systems are as follows:

1. Landau-Zener studies, ${ }^{5 h, i, m}$ in which we searched for potential surface crossings among

a. the entrance-channel ion-pair state in which an ETD anion donor and a positively charged model peptide initiate a collision and

b. states in which an electron has been transferred from the anion donor to a $3 \mathrm{~s}$ or $3 \mathrm{p}$ Rydberg orbital of a positive site, to an SS $\sigma^{*}$ orbital, or to an OCN $\pi^{*}$ orbital, showed that the cross sections for electron transfer to a Rydberg orbital of a positive site are ca. 10-100 times larger than for transfer to an SS $\sigma^{*}$ or an OCN $\pi^{*}$ orbital. So, attachment to a positive site is more likely, but direct attachment to the SS or OCN bond sites can occur $1-10 \%$ of the time.

2. Once an electron enters an SS $\sigma^{*}$ orbital, the SS bond is promptly cleaved; ${ }^{5 \mathrm{j}, 1}$ if it enters an $\mathrm{OCN} \pi^{*}$ orbital, a barrier must be overcome ${ }^{5 \mathrm{~m}}$ before $\mathrm{N}-\mathrm{C}_{\alpha}$ bond cleavage occurs.

3. Once an electron attaches to a $3 p$ or 3 s Rydberg orbital of a positive site, it can undergo transfer ${ }^{5 \mathrm{p}-\mathrm{s}}$ to an SS $\sigma^{*}$ or OCN $\pi^{*}$ orbital over ca. $15 \AA .{ }^{13}$ The exponential decay of the coupling strengths $H_{1,2}$ relating to the electron transfer is what limits the range of these electron-transfer processes. If the Rydberg orbital is already within $15 \AA$ of the SS $\sigma^{*}$ or OCN $\pi^{*}$ orbital when the electron attaches (i.e., in the ETD or ECD initial step), the electron need not reside in the Rydberg orbital for any appreciable time; instead, it can "shuttle" through ${ }^{16}$ the Rydberg orbital directly to the SS $\sigma^{*}$ or OCN $\pi^{*}$ orbital.

4. An electron initially attached to a $3 \mathrm{~s}$ or $3 \mathrm{p}$ orbital on one positive site can undergo transfer to another positive site $\mathrm{e}^{5 \mathrm{p}, \mathrm{q}, \mathrm{s}}$ but only if the two sites are within ca. $5 \AA$, so their Rydberg orbitals experience strong overlap. ${ }^{5 s}$ It does matter whether the two positive sites are of similar character (e.g., both protonated amines vs one protonated and one fixed-charge); the electron can only transfer if the electron binding strength of the acceptor site is higher than that of the donor. 


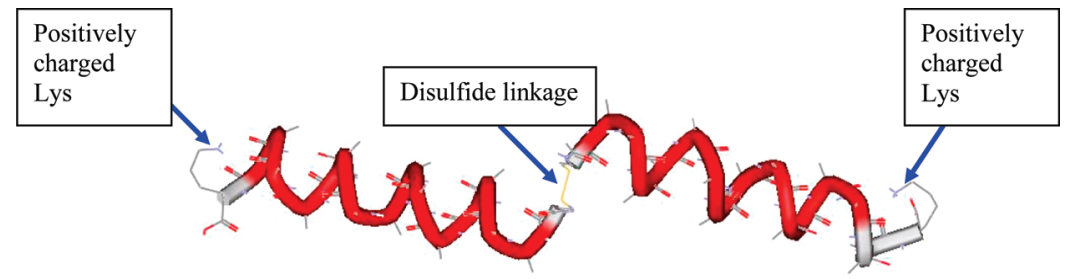

Figure 4. Assumed structure of doubly charged $\left(\mathrm{AcCA}_{n} \mathrm{~K}+\mathrm{M}\right)_{2}{ }^{2+}$ cations $(\mathrm{M}=\mathrm{H}$ or $\mathrm{Na})$ in the gas phase (redrawn from ref $\left.5 \mathrm{j}\right)$.

5. The coupling strengths $H_{1,2}$ connecting ground (3s) or lowenergy (3p) excited Rydberg states on positive sites to SS $\sigma^{*}$ or OCN $\pi^{*}$ orbitals, both of which decay exponentially with distance, do not differ from one another by more than an order of magnitude. ${ }^{5 r}$ However, the ground-state Rydberg orbitals on positive sites are known to be subject to prompt (ca. $10^{-9} \mathrm{~s}$ ) dissociation (e.g., a charge-neutralized $-\mathrm{NH}_{3}$ site loses a hydrogen atom or undergoes $\mathrm{C}-\mathrm{N}$ bond cleavage in this time scale) whereas most excited Rydberg states do not so dissociate. $^{14}$

6. The total cross sections for ECD and ETD fragment ion yield are large (often 10-100 $\AA^{2}$ ) and seem to scale as the square of the total charge $(Z)$ on the peptide. Our work showed ETD curve crossings leading to direct transfer from anion donor to $\mathrm{SS} \sigma^{*}$ or OCN $\pi^{*}$ orbitals occur at large distances and can also give large cross sections (e.g., $10 \AA^{2}$ and larger) if the probabilities of a surface hop are large. In fact, whenever crossings are involved in the electron attachment event, the cross sections can be shown to scale as $Z^{2}$, so this scaling law does not necessarily signal the involvement of high-energy Rydberg states. ETD cannot access as high-energy Rydberg orbitals as can ECD (because the anion donor's electron binding energy must be overcome), yet the fragmentation patterns of ETD and ECD are very similar. In electron-capture-induced dissociation ${ }^{15}$ (ECID), where an electron is transferred in a collision with ground-state sodium $\left(3 \mathrm{~s}^{1} ;{ }^{2} \mathrm{~S}\right)$ or cesium $\left(6 \mathrm{~s}^{1} ;{ }^{2} \mathrm{~S}\right)$ atoms and where even greater limitations exist on the energies of the Rydberg states of the peptide can be accessed, the fragmentation patterns are also quite similar. All of these findings suggest that initial electron attachment to high-energy Rydberg states may not be essential and are consistent with other steps being rate limiting for product-ion yields. In more recent work, ${ }^{16}$ we showed evidence suggesting that excited Rydberg states with principal quantum number $n<10$ are more central to the electron-transfer mechanisms.

We want to emphasize that we view all of the processes discussed above to be components of the so-called UtahWashington model because, regardless of whether an electron is initially captured at an SS or OCN site or at a positively charged site, and regardless of whether it moves from one positive site to another, the evidence suggests that it eventually has a good probability of ending up on an SS or OCN site and thus of cleaving an $\mathrm{S}-\mathrm{S}$ or $\mathrm{N}-\mathrm{C}_{\alpha}$ bond through the UW process.

In the present work, we focus on learning more about the intrapeptide electron-transfer events that allow an electron to migrate from an excited Rydberg orbital on a positive site ${ }^{17}$ to an SS $\sigma^{*}$ site or from a Rydberg orbital on one positive site to a Rydberg orbital on another site. Especially importantly, we expand on our earlier studies by considering many more Rydberg states, including those with higher principal quantum number. Because earlier efforts have made it clear that the vast majority of initial electron capture events (in ETD or ECD) involve electron attachment into a Rydberg orbital on one of the positively charged sites, we think it is important to devote more effort toward better characterizing the intrapeptide electron migration processes. In particular, in this paper we

(i) compare cases in which the positive and $\mathrm{SS} \sigma^{*}$ sites are separated by aliphatic or olefinic linkages of varying lengths as well as cases in which the two sites are separated by "vacuum" (i.e., no intervening molecular framework), and

(ii) examine the roles played by excited Rydberg orbitals with principal numbers higher than $n=3$ in effecting electron transfer.

In the latter component of this work, we are able to develop a useful analytical model that tells which Rydberg orbitals should be most effective in the electron-transfer process.

\section{Methods}

In the present studies of electron transfer from a Rydberg orbital on a positive site to the $\sigma^{*}$ orbital of a disulfide linkage, we first optimized the geometry of the parent model compound containing aliphatic $\mathrm{H}_{3} \mathrm{C}-\mathrm{S}-\mathrm{S}-\left(\mathrm{CH}_{2}\right)_{n}-\mathrm{NH}_{4}{ }^{+}$or olefinic $\mathrm{H}_{3} \mathrm{C}-\mathrm{S}-\mathrm{S}-(\mathrm{CH})_{n}-\mathrm{NH}_{4}{ }^{+}$spacers at the Hartree-Fock (HF) level with the distance between the nitrogen atom and the nearest sulfur atom held fixed and with the nitrogen atom and two sulfur atoms remaining collinear throughout the optimization. Next, the geometry optimization was refined using second-order Møller-Plesset perturbation theory (MP2). Then in subsequent calculations, other than elongating the SS bond whose cleavage we are studying, we retained these frozen geometries. We did so because (i) we were attempting to model the environment within a peptide or protein in which an $\mathrm{S}-\mathrm{S} \sigma^{*}$ orbital experiences a given (fixed) Coulomb stabilization and (ii) we wanted to model what took place immediately after vertical electron attachment to a Rydberg orbital on the positive site.

Because we wanted to extract information about the distance dependence of the electron-transfer rates, it was important to have the distance $R_{\mathrm{NS}}$ from the $\mathrm{S}-\mathrm{S}$ bond to the $\mathrm{NH}_{4}{ }^{+}$site to vary from one model system to another. To achieve this, we carried out such calculations with varying number of aliphatic or olefinic spacer groups in which $R_{\mathrm{NS}}$ ranged from ca. 4 to ca. $11 \AA$ A. In addition, to explore what happens when the positive and SS sites are separated by vacuum rather than aliphatic or olefinic linkages, we carried out analogous calculations on model systems $\mathrm{H}_{3} \mathrm{C}-\mathrm{SS}-\mathrm{CH}_{3} \cdots \mathrm{NH}_{4}{ }^{+}$consisting of a dimethyl disulfide molecule separated from an ammonium cation by a distance $R_{\mathrm{NS}}$; we chose values of $R_{\mathrm{NS}}$ similar to those pertinent to the $\mathrm{H}_{3} \mathrm{C}-\mathrm{S}-\mathrm{S}-\left(\mathrm{CH}_{2}\right)_{n}-\mathrm{NH}_{4}{ }^{+}$and $\mathrm{H}_{3} \mathrm{C}-\mathrm{S}-\mathrm{S}-(\mathrm{CH})_{n}-\mathrm{NH}_{4}{ }^{+}$ model compounds.

The three families of "spacers", aliphatic, olefinic, and vacuum, used in our calculations serve different purposes:

1. The aliphatic spacers are qualitatively representative of the saturated bonding motifs that connect $\mathrm{SS}$ or $\mathrm{N}-\mathrm{C}_{\alpha}$ bonds, along a peptide's backbone and further along a side chain, to a Rydberg orbital on one of the peptide's positively charges sites. The $H_{1,2}$ coupling strengths obtained with such spacers are thus most appropriate for describing through-bond electron transfer. 
2. The vacuum spacer (at various distances) is used to treat through-space electron-transfer cases.

3. The olefinic spacers are probably not representative of bonding environments near SS or $\mathrm{N}-\mathrm{C}_{\alpha}$ bonds in peptides since peptide backbones do not contain extended $\pi$-bonding networks, although some amino acids' side chains do. Thus, our results on the olefinic spacer systems are probably of more importance for understanding electron transfer in molecular frameworks other than polypeptides, but we decided to include their treatment to broaden the potential impact of this work.

Special atomic basis functions had to be employed to describe the Rydberg states on the positively charged groups. In particular, three sets of $\mathrm{s}$ and $\mathrm{p}$ extra-diffuse Rydgerg-type basis functions ${ }^{18}$ centered on the protonated nitrogen atom were added to the aug-cc-pVDZ basis sets ${ }^{19}$ used for all the other atoms. This kind of basis was shown earlier ${ }^{18}$ to be capable of reproducing the energies of several low-energy Rydberg states of nitrogen-centered radicals.

In evaluating the potential energy surfaces pertinent to our studies of electron transfer from a Rydberg orbital on a positive site to the SS $\sigma^{*}$ orbital, we had to evaluate the energies of the ground and several excited Rydberg states as well as the energy of the state in which an electron occupies the Coulomb-stabilized SS $\sigma^{*}$ orbital. We had to converge HF calculations on several such states and then perform MP2-level correlated calculations on each state.

The results of such calculations on many electronic states are families of Born-Oppenheimer adiabatic potential energy surfaces. To probe for conditions under which electron transfer should be facile, we search for geometries near which pairs of such curves undergo avoided crossings. In the figures shown later in this paper, some pairs of curves appear to cross. However, in reality, all of the curves we show undergo avoided crossings. If the couplings between a given pair of states are very weak, the "avoidance" may be too small to see at the resolution of the figure, but it is still present.

Finally, we note that all calculations were performed using the Gaussian 03 suite of programs, ${ }^{20}$ and the three-dimensional plots of the molecular orbitals were generated with the MOLDEN program. ${ }^{21}$

\section{Results}

A. Intramolecular Electron Transfer Involving Aliphatic, Olefinic, and Vacuum Linkers. In Figure 5, we show the energy profiles obtained in our computations for aliphatic model compounds ${ }^{+} \mathrm{H}_{3} \mathrm{~N}-\left(\mathrm{CH}_{2}\right)_{n}-\mathrm{SS}-\mathrm{CH}_{3}$ for $n=2$ and 4 , in which cases the distances from the nitrogen atom to the nearest sulfur atom are 4.1 and $6.6 \AA$, respectively. We also show the corresponding data for the olefinic compounds ${ }^{+} \mathrm{NH}_{3} \mathrm{~N}-(\mathrm{CH})_{n}-$ $\mathrm{SS}-\mathrm{CH}_{3}$ for $n=2,4,6$, and 8 where the $\mathrm{S}-\mathrm{N}$ distances are 4.1, 6.5, 9.0, and $11.4 \AA$, respectively. We do not display data for aliphatic ${ }^{+} \mathrm{H}_{3} \mathrm{~N}-\left(\mathrm{CH}_{2}\right)_{n}-\mathrm{SS}-\mathrm{CH}_{3}$ species with $n>4$ because, for such species, the SS $\sigma^{*}$-attached state's potential curve does not cross any of the Rydberg states' curves at $S-S$ bond lengths that are thermally accessible. Also included in Figure 5 are the $\mathrm{H}_{1,2}$ coupling strengths connecting the $\mathrm{SS} \sigma^{*}$ attached and various Rydberg-attached states.

The first thing to notice in Figure 5 is that the "shapes" (i.e., variation with SS bond length) and relative energies of the parent and Rydberg-attached states' curves are essentially identical for all of the model systems. That is, all of these curves are nearly parallel to one another as functions of the SS bond length. This suggests that the diabatic Rydberg states are influenced very little by the presence of proximal aliphatic or olefinic linkages.
In fact, as shown in Figure 6, essentially these same parent and Rydberg-state energy profiles result even if the protonated amine and disulfide sites are separated by vacuum (at S-to- $\mathrm{N}$ distances characteristic of the ${ }^{+} \mathrm{H}_{3} \mathrm{~N}-\left(\mathrm{CH}_{2}\right)_{2}-\mathrm{SS}-\mathrm{CH}_{3}$ and ${ }^{+} \mathrm{H}_{3} \mathrm{~N}-$ $\left(\mathrm{CH}_{2}\right)_{4}-\mathrm{SS}-\mathrm{CH}_{3}$ species, respectively) rather than by olefinic or aliphatic linkages.

In contrast to the behavior of the cation and Rydberg states, the relative energy of the SS $\sigma^{*}$-attached state is seen in Figures 5 and 6 to depend strongly both on the nature of the linkage (i.e., aliphatic, olefinic, or vacuum) and on the distance $\left(R_{\mathrm{NS}}\right)$ from the nitrogen atom to the closest sulfur atom. However, the "shape" of the SS $\sigma^{*}$-attached curve (i.e., its dependence on $R_{\mathrm{NS}}$ aside from its asymptotic energy) varies little from case to case shown in Figures 5 and 6. It is, of course, not surprising that the asymptotic energy of the $\sigma^{*}$-attached state should depend strongly on $R_{\mathrm{NS}}$ because the Coulomb stabilization experienced by the electron occupying the SS $\sigma^{*}$ orbital varies as $14.4 \mathrm{eV} / R_{\mathrm{NS}}$. Specifically, for the aliphatic-linkage cases, the large $R_{\mathrm{SS}}$ asymptote of the $\sigma^{*}$-attached state can be accurately estimated (Figure 5a,b and Figure 6) by taking the intrinsic electron binding energy of the ${ }^{\mathrm{S}} \mathrm{S}-\mathrm{R}$ radical (ca. $2 \mathrm{eV}$ with the basis sets and level of correlation used in our work) and adding in the Coulomb stabilization energy $(14.4 \mathrm{eV} / 4.1 \AA=3.8 \mathrm{eV}$ for the $-\left(\mathrm{CH}_{2}\right)_{2}-$ case or $14.4 \mathrm{eV} / 6.6 \AA=2.2 \mathrm{eV}$ for the $-\left(\mathrm{CH}_{2}\right)_{4}-$ case). These estimates also work reasonably well for the cases (Figure 6) in which there is only vacuum between the two sites, which suggests the $-\mathrm{CH}_{2}-$ spacers have little intrinsic influence.

However, these Coulomb stabilization estimates do not work when olefinic linkages are present (Figure $5 \mathrm{c}-\mathrm{f}$ ). In fact, the relative energies of the Rydberg- and SS $\sigma^{*}$-attached states for the (olefinic) ${ }^{+} \mathrm{H}_{3} \mathrm{~N}-(\mathrm{CH})_{4}-\mathrm{SS}-\mathrm{CH}_{3},{ }^{+} \mathrm{H}_{3} \mathrm{~N}-(\mathrm{CH})_{6}-\mathrm{SS}-\mathrm{CH}_{3}$, and ${ }^{+} \mathrm{H}_{3} \mathrm{~N}-(\mathrm{CH})_{8}-\mathrm{SS}-\mathrm{CH}_{3}$ cases are nearly identical to those for the (olefinic) ${ }^{+} \mathrm{H}_{3} \mathrm{~N}-(\mathrm{CH})_{2}-\mathrm{SS}-\mathrm{CH}_{3}$ and the (aliphatic) ${ }^{+} \mathrm{H}_{3} \mathrm{~N}-\left(\mathrm{CH}_{2}\right)_{2}-\mathrm{SS}-\mathrm{CH}_{3}$ cases. This suggests that the olefinic linkages are able to delocalize the negative charge ${ }^{22}$ of the SS $\sigma^{*}$-attached state closer to the stabilizing positively charged ${ }^{+} \mathrm{H}_{3} \mathrm{~N}-\mathrm{R}$ site. Although these findings may not be of direct relevance to through-bond electron transfer in peptides (because the SS and OCN bond sites are not connected to positively charged sites by olefinic linkages), they will likely be of interest to workers studying electron transfer in other cases.

The $H_{1,2}$ coupling strengths shown in Figures 5 and 6 display trends that we will discuss further in subsection $\mathrm{C}$ where we derive analytical models for how these couplings should depend upon the distance between the SS and Rydberg sites and upon the $\mathrm{n}$ quantum number of the Rydberg orbital. For now, it is worth noting the following:

1. The $H_{1,2}$ values of the $3 p$ and $3 \mathrm{~s}$ Rydberg states are of similar magnitude, as expected, and are similar in size to those we found in our earlier studies.

2. Among the $3 p$ states, there is one (that in which the $3 p$ Rydberg orbital is aligned perpendicular to the SS $\sigma^{*}$ orbital) that has an $H_{1,2}$ value smaller than those of the other two $3 \mathrm{p}$ states. Which $3 \mathrm{p}$ state has the smallest $H_{1,2}$ value varies from case to case because the orientation of the molecule within the Cartesian coordinate system varies.

3. The $\mathrm{H}_{1,2}$ values of the $4 \mathrm{~s}$ state are usually smaller but not much less than those of the $3 \mathrm{~s}$ and $3 \mathrm{p}$ states. This observation is important and is in line with the analytical model developed later for how the $H_{1,2}$ values should vary with the n quantum number of the Rydberg orbital.

4. When olefinic linkages are present, there is not much decay in the magnitudes of the $H_{1,2}$ values with the $\mathrm{N}-\mathrm{S}$ distance, 
(a)

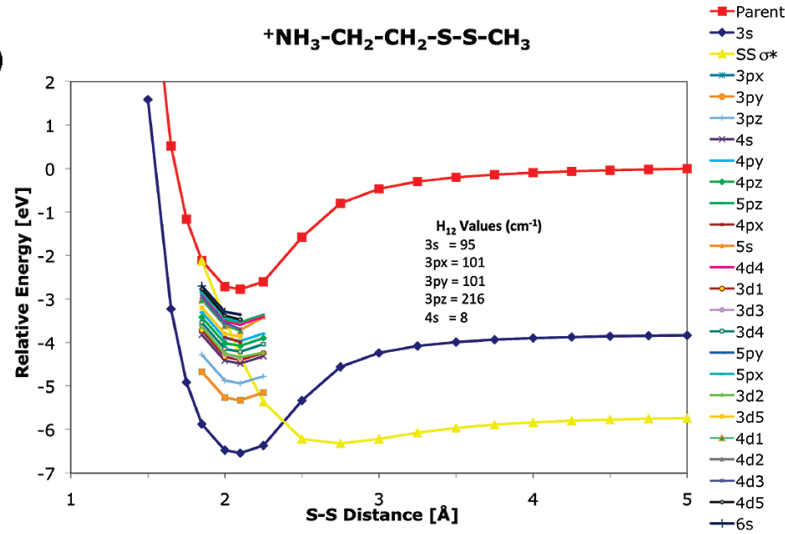

(b)

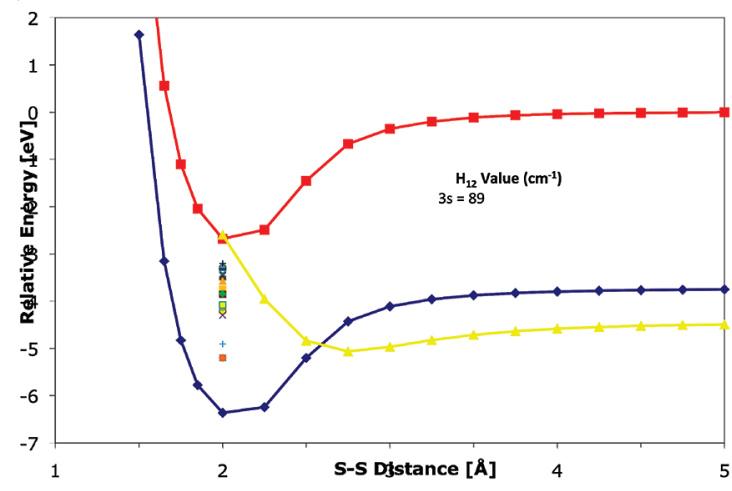

(c)

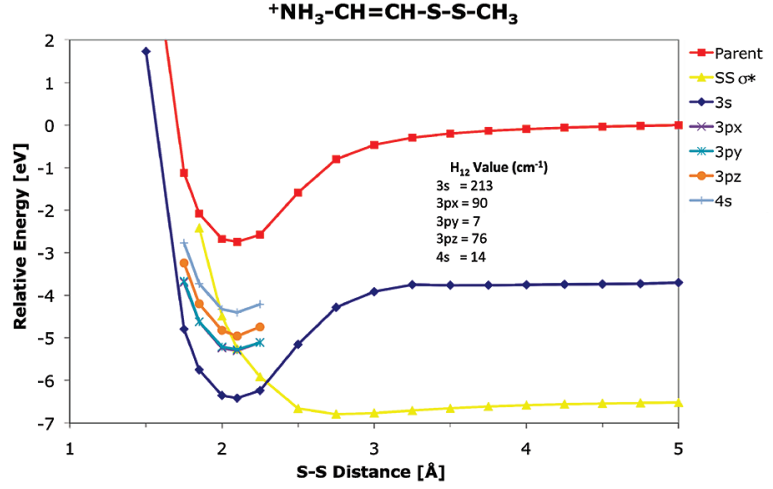

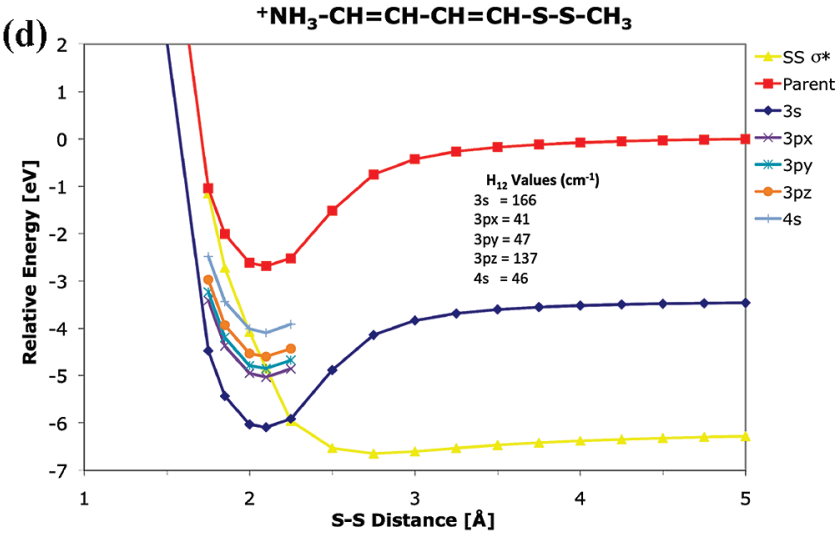

(e) $\quad+\mathrm{NH}_{3}-\mathrm{CH}=\mathrm{CH}-\mathrm{CH}=\mathrm{CH}-\mathrm{CH}=\mathrm{CH}-\mathrm{S}-\mathrm{S}-\mathrm{CH}_{3}$

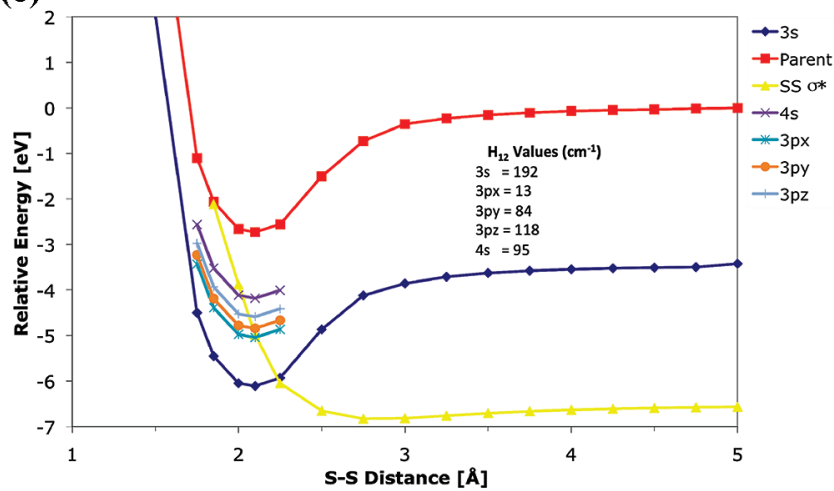

(f)

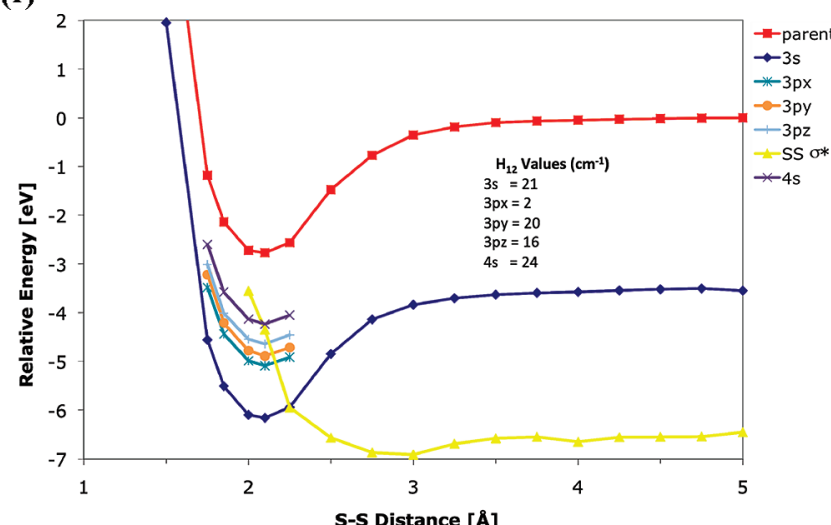

Figure 5. Energies of the parent cation (red), ground 3s Rydberg-attached (blue), and SS $\sigma^{*}$-attached (yellow) states of model compounds containing aliphatic (a), (b) or olefinic (c)-(f) linkages between protonated amine and disulfide bond sites, as functions of the SS bond length. Also shown in the top figure are energies of 3p, 3d, 4s, 4p, 4d, 5s, and 5p Rydberg-attached states. In the second through sixth figures, the energies of these same excited Rydberg states are shown but only over a narrow range of SS bond lengths near the equilibrium geometry of the parent compound. Also shown are the $H_{1,2}$ coupling matrix elements between the SS $\sigma^{*}$ and $3 \mathrm{~s}, 3 \mathrm{p}$, and $4 \mathrm{~s}$ Rydberg states.

which is consistent with the delocalization of the $\mathrm{SS} \sigma^{*}$-attached electron noted above.

B. Exponential Decay of Coupling with Distance for a Specified Rydberg State. Another issue that is important to address is how the coupling between the Rydberg- and SS $\sigma^{*}$ attached states depends on the distance $R_{\mathrm{NS}}$ between the positive and SS sites. In Figure 7 we show how the $H_{1,2}$ coupling matrix elements for the ${ }^{+} \mathrm{H}_{3} \mathrm{~N}-\left(\mathrm{CH}_{2}\right)_{n}-\mathrm{SS}-\mathrm{CH}_{3}$ model compounds containing aliphatic spacer units decays with distance for the $3 s$ and $3 p$ Rydberg states. As in other studies we carried out, these couplings appear to decay exponentially with distance, as expected. By extrapolating such plots to longer distances, we have been able to estimate that the critical $H_{1,2}$ value of ca.
$0.3 \mathrm{~cm}^{-1}$ (at which the rate of interpeptide electron transfer falls below $10^{6} \mathrm{~s}^{-1}$ ) will be reached at distances of $15-20 \AA$.

However, as the data shown in Figures 5 and 6 show, there are many Rydberg states that might cross the repulsive SS $\sigma^{*}$ attached state's curve near the equilibrium geometry of the parent ion. Although our experience (e.g., on the $3 \mathrm{~s}$ and $3 \mathrm{p}$ Rydberg states as illustrated in Figure 7) and fundamental considerations of how orbital depend on the radial position of the electron suggest that orbital overlaps and, by inference, $H_{1,2}$ values can be expected to decay exponentially with distance, it is also important to establish how the $H_{1,2}$ couplings depend upon the Rydberg orbital's $n$ quantum number. This is important because, as Figures 5 and 6 make clear, there are many Rydberg 
N S Distance of 4.1 A: $\quad \mathrm{H}_{3} \mathrm{~N}^{+}-\mathrm{CH}_{3} \quad \mathrm{H}_{3} \mathrm{C}-\mathrm{S}-\mathrm{S}-\mathrm{CH}_{3}$

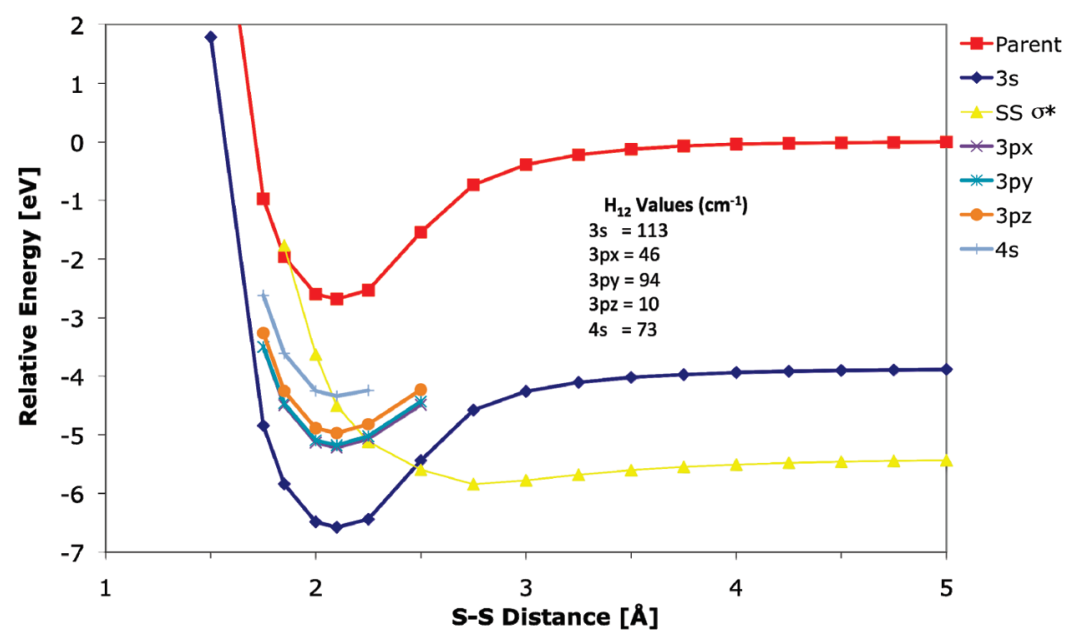

N S Distance of $6.5 \AA$ : $\quad \mathrm{H}_{3} \mathrm{~N}^{+}-\mathrm{CH}_{3} \quad \mathrm{H}_{3} \mathrm{C}-\mathrm{S}-\mathrm{S}-\mathrm{CH}_{3}$

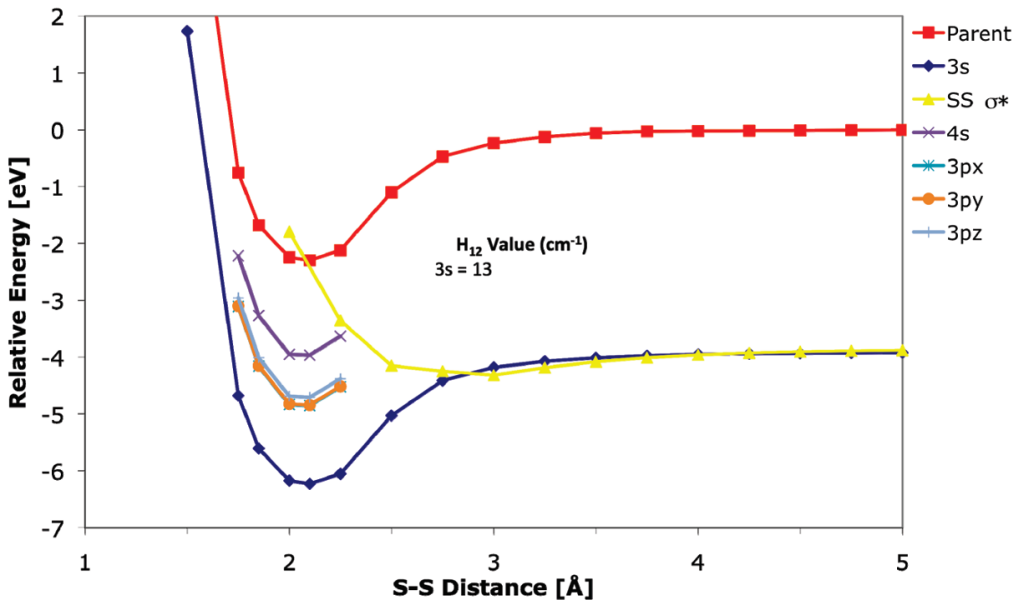

Figure 6. Energies of the parent cation (red), ground 3s Rydberg-attached (blue), and SS $\sigma^{*}$-attached (yellow) states of model compounds containing only vacuum between protonated amine and disulfide bond sites. Also shown are the $H_{1,2}$ coupling matrix elements between the SS $\sigma^{*}$ and $3 \mathrm{~s}, 3 \mathrm{p}$, and 4s Rydberg states.

states that may be accessible to electron attachment and that may subsequently be energetically and geometrically well placed to effect electron transfer to an SS $\sigma^{*}$ orbital. We need to know how the couplings of such states vary with $n$ and with distance. One way to approach this problem is to attempt to explicitly compute (i.e., as we have done for the $3 \mathrm{~s}$ and $3 \mathrm{p}$ Rydberg states whose couplings are shown in Figure 7) these $H_{1,2}$ data using ab initio methods. However, as Figure 5 makes clear, there is often a plethora of Rydberg states to be considered. Because the evaluation of $H_{1,2}$ couplings is very tedious (i.e., it involves evaluating the energies of the two adiabatic states that undergo an avoided crossing at a very finely spaced grid of geometries), it would be extremely taxing to pursue this route. Therefore, in the following section, we opt to derive an approximate framework in terms of which we can estimate $H_{1,2}$ values for Rydberg-SS $\sigma^{*}$ couplings involving Rydberg states having $n>$ 3 in terms of the (far fewer) ab initio $H_{1,2}$ values we compute for states having $n=3$. That is, we derive a scaling formula giving the $n$ dependence of $H_{1,2}$.

C. Analytical Model for How Coupling Strength Depends on Rydberg Orbitals' Principal Quantum Number and Radial Extent. First, it helps to gain some perspective about how radially extended various Rydberg orbitals are and to what extent they can overlap an SS $\sigma^{*}$ or OCN $\pi^{*}$ orbital at various intersite distances. In Figure 8 we show, for $\mathrm{NH}_{4}$, the $3 \mathrm{~s}, 3 \mathrm{p}$, 3d, 4s, 4p, and 5s Rydberg orbitals all drawn to the same scale and using an outermost contour within which $60 \%$ of that orbital's electron density resides (i.e., $40 \%$ of the electron density resides at larger distances). Within each of these Rydberg orbitals, one can see the underlying $\mathrm{NH}_{4}{ }^{+}$cation's van der Waals surface. Comparing the van der Waals size of $\mathrm{NH}_{4}{ }^{+}$to the Rydberg orbitals' outer contour gives some idea of how large these orbitals are. Rydberg orbitals on protonated amines $\mathrm{R}-\mathrm{NH}_{3}{ }^{+}$are very similar in radial extent to those of $\mathrm{NH}_{4}$ although, of course, the lower symmetry causes degeneracies that occur for $\mathrm{NH}_{4}$ to be removed. In Figure 8, we also show the $3 \mathrm{~s}$ and $4 \mathrm{~s}$ orbitals of $\mathrm{H}_{3} \mathrm{C}-\mathrm{NH}_{3}$ to illustrate that the qualitative shapes and sizes of the Rydberg orbitals are altered by alkyl-group substitution.

In the figure showing the ground 3s Rydberg orbital of $\mathrm{NH}_{4}$, we also show a qualitative drawing of an SS $\sigma^{*}$ orbital shown at three distances from the center of the Rydberg orbital. At the distance for which the orbital is shown in green, the $\sigma^{*}$ orbital has much of its probability amplitude within the radial region where the 3s Rydberg orbital has its major amplitude, so the overlap of the $\sigma^{*}$ and Rydberg orbitals should be near its maximum. At the longer distances where the orbital is shown in blue or red, the $\sigma^{*}$ orbital is further from the center of the 

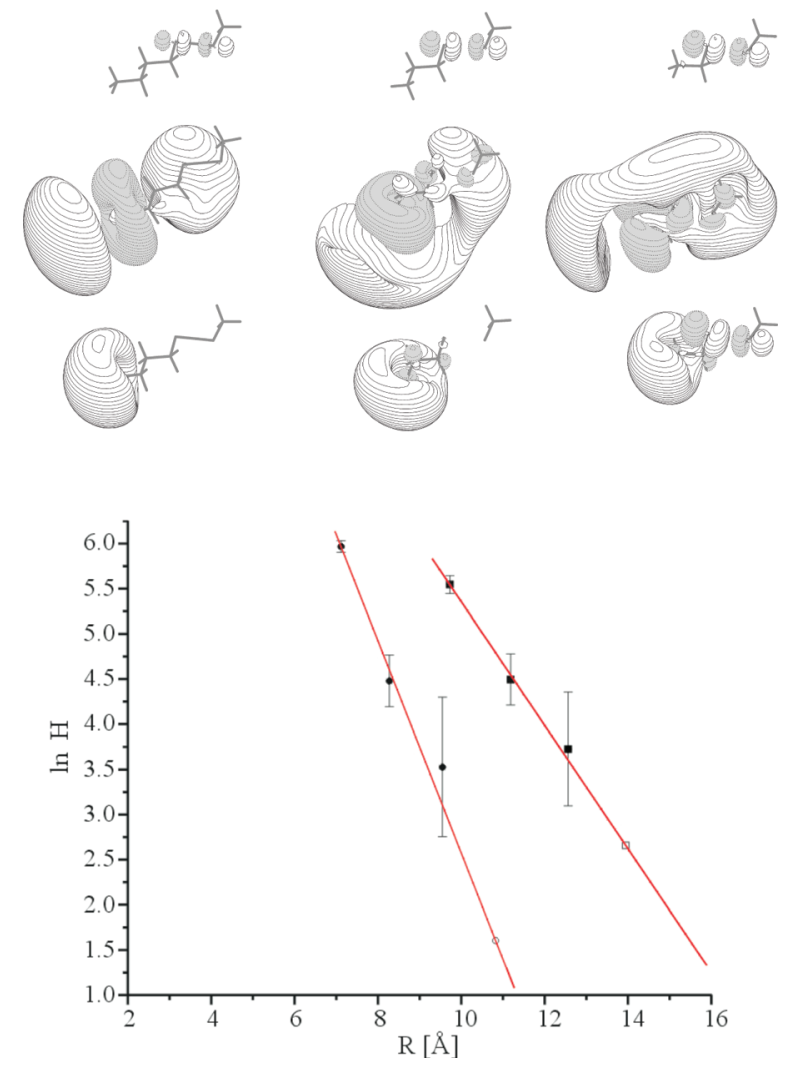

Figure 7. Plots of $\ln H_{1,2}\left(\mathrm{~cm}^{-1}\right)$ vs distance $R(\AA)$ between the center of the SS bond and the center of charge of the $3 \mathrm{~s}$ ground (left line) and $3 p$ excited (right line) Rydberg orbitals for the ${ }^{+} \mathrm{H}_{3} \mathrm{~N}-\left(\mathrm{CH}_{2}\right)_{n}-$ $\mathrm{S}-\mathrm{S}-\mathrm{CH}_{3}$ model compounds having $n=1,2$, and 3. Also shown (top) are the structures of the ${ }^{+} \mathrm{H}_{3} \mathrm{~N}-\left(\mathrm{CH}_{2}\right)_{n}-\mathrm{S}-\mathrm{S}-\mathrm{CH}_{3}$ model systems and their SS $\sigma^{*}$ (top), 3p (middle), and 3s (bottom) Rydberg orbitals (appears as Figures 2 and 4 in ref $5 p$, reproduced with permission; copyright 2008, Elsevier).

Rydberg orbital, so the overlap values should be smaller for the blue and even smaller for the red case. The decrease in overlap, and thus in $H_{1,2}$ coupling strength, from green to blue to red is expected to follow an exponential form as a function of $R_{\mathrm{NS}}$ the nitrogen-to-sulfur distance as shown in Figure 7. Similar trends are expected for the distance dependence of the overlap of an OCN $\pi^{*}$ orbital with a Rydberg orbital.

In Figure 8, we also show an SS $\sigma^{*}$ orbital overlapping other (4s, 4p, and 5s) Rydberg orbitals at distances characteristic of where these Rydberg orbitals have major probability amplitude. ${ }^{23}$ It is at such distances that these $\sigma^{*}$-Rydberg orbital overlaps are expected to be largest (hence the green color in Figure 8). As noted above, the overlap of an $\mathrm{SS} \sigma^{*}$ (or OCN $\pi^{*}$ ) orbital with a given Rydberg orbital is expected to decay exponentially with $R_{\mathrm{NS}}$. This knowledge is important for us to use in estimating rates of interpeptide electron transfer. However, it is also important for us to know how the overlaps vary as the principal quantum number (and hence the radial location of maximum probability amplitude) of the Rydberg orbital varies, and this is the subject to which we now turn our attention.

We begin by using the fact that Rydberg orbitals have hydrogen-like radial forms ${ }^{24}$ at large distances $r$

$$
R_{n}(r)=N r^{n-1} \mathrm{e}^{-Z r / n a_{0}}
$$

where $a_{0}$ is the Bohr unit of length $(0.529 \AA), r$ is the distance of the electron from the positive site, $n$ is the principal quantum
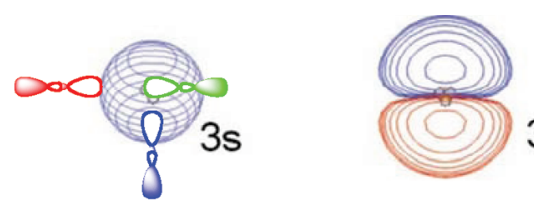

$3 p$
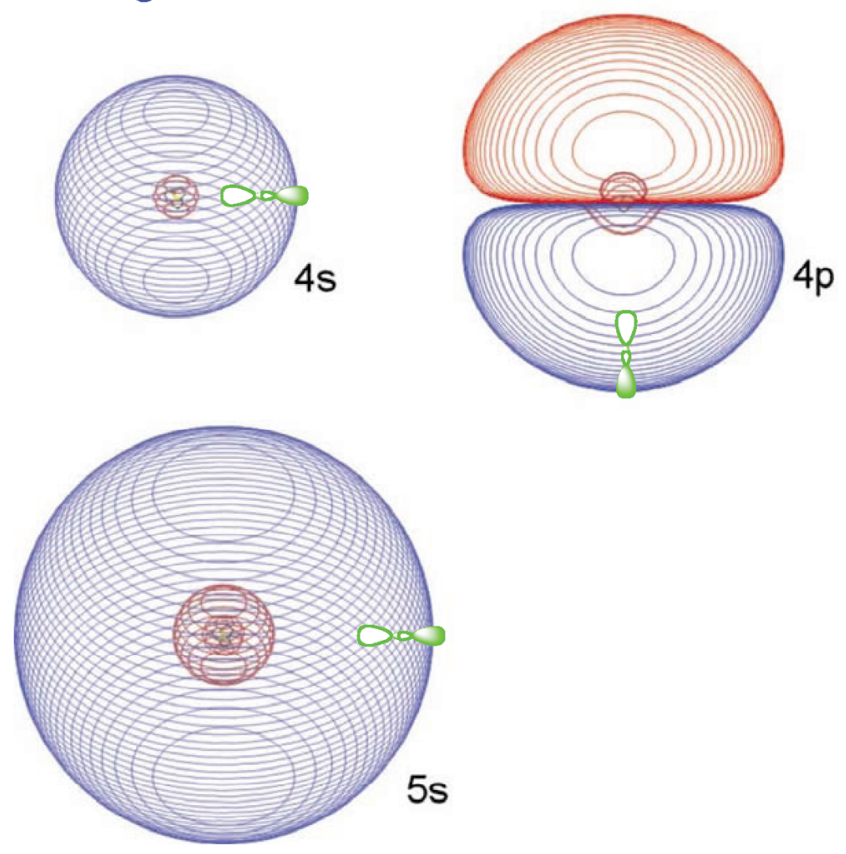

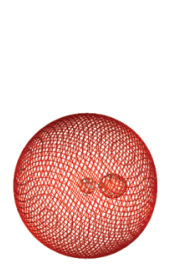

$3 \mathrm{~s}$

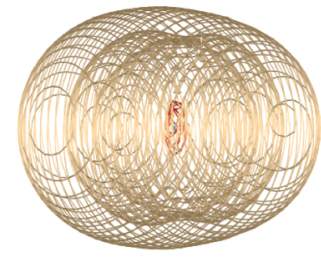

$4 \mathrm{~s}$
Figure 8. Plots of $3 \mathrm{~s}, 3 \mathrm{p}, 3 \mathrm{~d}, 4 \mathrm{~s}$, and $5 \mathrm{~s}$ Rydberg orbitals of $\mathrm{NH}_{4}$ with the outermost contour containing $60 \%$ of the electron density of that orbital. Also shown are qualitative illustrations of SS $\sigma^{*}$ orbitals at positions producing maximum (green), intermediate (blue), and low (red) overlap with some of the Rydberg orbitals. In the bottom row the $3 \mathrm{~s}$ and $4 \mathrm{~s}$ Rydberg orbitals of $\mathrm{H}_{3} \mathrm{C}-\mathrm{NH}_{3}$ are shown also at $60 \%$ outermost contours.

number, and $Z$ is the charge on the ion. From this, one can obtain the following expressions for the average radial size $\langle r\rangle$ and for the average $\left\langle r^{2}\right\rangle$ in terms of $n$ and $Z$ :

$$
\begin{gathered}
\langle r\rangle=\frac{n\left(n+\frac{1}{2}\right) a_{0}}{Z} \\
\left\langle r^{2}\right\rangle=\frac{\left(n+\frac{1}{2}\right)(n+1) n^{2} a_{0}^{2}}{Z^{2}}
\end{gathered}
$$

These expressions allow us to introduce the "thickness" $T$ of the radial probability density $P(r)=r^{2}\left|R_{n}(r)\right|^{2}$ of a Rydberg orbital defined as the root-mean-square range of its radial distribution: 


$$
T=\sqrt{\left\langle r^{2}\right\rangle-\langle r\rangle^{2}}=\frac{\sqrt{2 n+1}}{2} \frac{n a_{0}}{Z}
$$

In eqs 6 and 8, one sees that the radial sizes of Rydberg orbitals grow as $n^{2}$, but their radial thickness grows less rapidly as $n(2 n$ $+1)^{1 / 2}$. So, as the sizes $\langle r\rangle$ of the radial probability distributions of Rydberg orbitals grow with $n$, their thicknesses decrease as a fraction of their radial extent (i.e., $T /\langle r\rangle$ decreases as $n$ increases). In Figure 9 we show a qualitative depiction of the radial probability density of a Rydberg orbital (ignoring its oscillatory behavior ${ }^{24}$ at smaller-r), its average radial $\operatorname{size}^{25}\langle r\rangle$, and its thickness $T$.

To conceptualize the magnitude of the overlap ${ }^{26}$ (which we will assume to be proportional to the $H_{1,2}$ coupling strength) of a Rydberg orbital with an SS $\sigma^{*}$ or an amide $\pi^{*}$ orbital, let us think of a Rydberg orbital as having most of its electron density within a spherical shell of radius $\langle r\rangle$ and thickness T. Using eqs 6 and 8, we can show (for $T<\langle r\rangle$ ) this shell to have a volume of

$$
V_{n}=4 \pi\langle r\rangle^{2} T=4 \pi \frac{n^{3}\left(n+\frac{1}{2}\right)^{2} \sqrt{2 n+1} a_{0}^{3}}{2 Z^{3}}
$$

An SS $\sigma^{*}$ or an amide $\pi^{*}$ orbital has a volume that can be expressed as $V_{\text {bond }}={ }^{4} / 3 \pi\left(x a_{0}\right)^{3}$, where the dimensionless variable $x$ can be used to characterize the radial extent of either of these valence-size orbitals and is expected to be of the order of 5-10 (i.e., the valence orbitals are assumed to be smaller than the Rydberg orbitals and ca. 5-10 $a_{0}$ in radial extent).

Now, consider one of these antibonding orbitals fully inserted into the shell of thickness $T$ of a Rydberg orbital as illustrated by the green orbitals in Figure 8. That is, we assume that the valence orbital is small enough to have essentially its entire volume residing within the region where the Rydberg orbital has most of its amplitude-between $\langle r\rangle-T / 2$ and $\langle r\rangle+T / 2$ (see Figure 9). Finally, let us assume that the electron density within the Rydberg and valence orbitals having volumes $V_{n}$ and $V_{\text {bond }}$, respectively, can be approximated as spatially uniform within the shell of thickness $T$. For example, in Figure 9, we assume that the entire probability density of the Rydberg orbital is uniformly distributed within the shell of thickness $T$ outlined by the green lines. Within each volume, the respective wave functions can therefore approximated by $\Psi_{n}(\mathbf{r})=\left(1 / V_{n}\right)^{1 / 2}$ and $\Psi_{\text {bond }}(\mathbf{r})=\left(1 / V_{\text {bond }}\right)^{1 / 2}$. The $H_{1,2}$ coupling should scale with $n$ in the same manner as the overlap integral $(S)$ between these two wave functions. The overlap can be evaluated by integrating the product of the two wave functions $\Psi_{n}(\mathbf{r})$ and $\Psi_{\text {bond }}(\mathbf{r})$ over the volume $V_{\text {bond }}$ that they share:

$$
\begin{aligned}
S=\int_{V_{\text {bond }}} \frac{1}{V_{\text {bond }}^{1 / 2}} \frac{1}{V_{n}^{1 / 2}} \mathrm{~d}^{3} r & =\frac{V_{\text {bond }}^{1 / 2}}{V_{n}^{1 / 2}}= \\
& \sqrt{\frac{2 x^{3} Z^{3}}{3 n^{3}\left(n+\frac{1}{2}\right)^{2} \sqrt{2 n+1}}}
\end{aligned}
$$

Having developed an analytical (albeit qualitative ${ }^{27}$ ) model for how the valence-Rydberg orbital overlaps vary with $n$, we are now in a position to estimate how the rates of intrapeptide

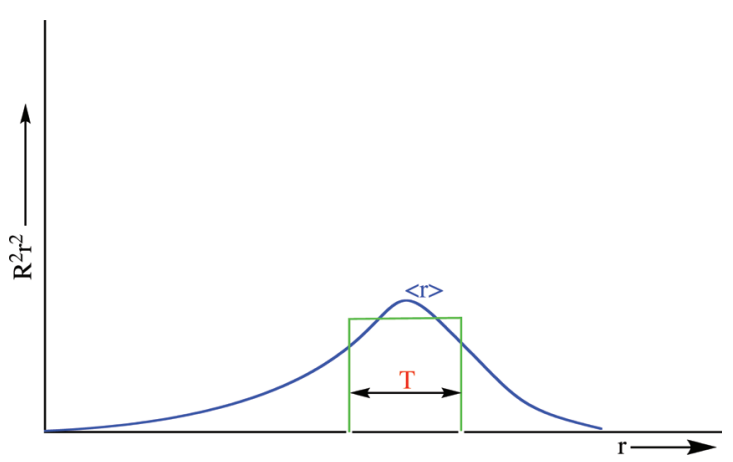

Figure 9. Qualitative depiction (blue curve) of the radial probability density $\left.|R|\right|^{2} r^{2}$ of a Rydberg orbital as a function of distance $r$ from the positive center of the orbital to the electron. Also shown is the average radial size $\langle r\rangle$ and the thickness $T$ of the orbital.

TABLE 1: Radial Sizes and Maximum Through-Space Electron-Transfer Rates for Transfer from Rydberg Orbitals of Various Principal Quantum Number $\boldsymbol{n}$ to a Valence SS $\boldsymbol{\sigma}^{*}$ or OCN $\pi^{*}$ Orbital

\begin{tabular}{ccc}
\hline $\begin{array}{c}\text { principal quantum } \\
\text { number }\end{array}$ & $\begin{array}{c}\text { radial size } \\
(\AA) \text { (̊) for } Z=1\end{array}$ & $\begin{array}{c}\text { maximum rate }^{b} \\
\left(\mathrm{~s}^{-1}\right) \text { for } Z=1\end{array}$ \\
\hline 3 & 5.5 & $10^{12}$ \\
4 & 9.5 & $2 \times 10^{11}$ \\
5 & 14.5 & $7 \times 10^{10}$ \\
6 & 20.6 & $3 \times 10^{10}$ \\
7 & 27.8 & $1 \times 10^{10}$ \\
8 & 36.0 & $6 \times 10^{9}$ \\
10 & 55.5 & $2 \times 10^{9}$ \\
20 & 217 & $4 \times 10^{7}$
\end{tabular}

${ }^{a}$ According to eq $6,\langle r\rangle$ should scale with $Z$ as $Z^{-1}$. ${ }^{b}$ According to eq 10 and the fact that the rate depends on $S^{2}$, the rate should scale with $Z$ as $Z^{3}$.

electron transfer depend upon the $n$ quantum number (and hence the radial size) of the Rydberg orbital involved. Specifically, we

(i) use the data we obtained earlier on $H_{1,2}$ values for $3 \mathrm{~s}$ and 3 p Rydberg orbitals coupling to SS $\sigma^{*}$ or OCN $\pi^{*}$ orbitals where $H_{1,2}$ values in the $100-300 \mathrm{~cm}^{-1}$ range were obtained when the valence orbital had strong overlap ${ }^{28}$ with the Rydberg orbital,

(ii) assume that eq 4 can be used to estimate the rates of electron transfer once $H_{1,2}$ is known, and

(iii) assume that $H_{1,2}$ values scale with $\mathrm{n}$ as in eq 10 for the overlap integral.

We can then approximate the maximum rates ${ }^{29}$ of electron transfer from Rydberg orbitals having $n=4,5,6$, etc. in terms of the maximum rates (ca. $10^{12} \mathrm{~s}^{-1}$ ) observed for the $3 \mathrm{~s}$ and $3 \mathrm{p}$ Rydberg orbitals using the scaling law of eq 10 and the fact that the rates are proportional to the square of $H_{1.2}$ :

$$
\text { rate }_{n}=\frac{3^{3}(3+1 / 2)^{2} \sqrt{2(3)+1}}{n^{3}(n+1 / 2)^{2} \sqrt{2 n+1}} \times 10^{12} \mathrm{~s}^{-1}
$$

In Table 1, we show these estimated maximum rates as well as the radial sizes $\langle r\rangle$ of the Rydberg orbitals for $n$ ranging from 3 through 20.

Although these estimates are probably rather rough, they suggest that the maximum (i.e., when the SS or OCN valence orbital is fully subsumed by the Rydberg orbital) rates of intrapeptide electron transfer will decay slowly as $n$ increases and will remain considerably in excess of the ca. $10^{6} \mathrm{~s}^{-1}$ cutoff 


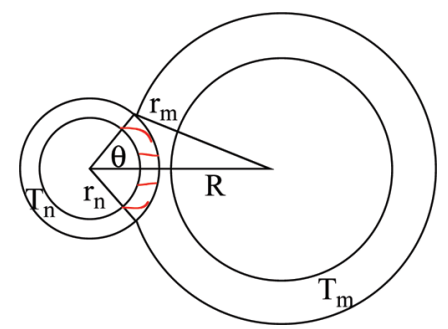

Figure 10. Qualitative depiction of two s-symmetry Rydberg orbitals having sizes characterized by radii $r_{n}$ and $r_{m}$ with thicknesses $T_{n}$ and $T_{m}$ separated by a distance $R<r_{n}+r_{m}$.

beyond which electron transfer cannot compete with radiationless relaxation within the Rydberg manifold, even for Rydberg orbitals having $n$ values as high as 20 . Indeed the $H_{1,2}$ values shown in Figures 5 and 6 for $n=3$ and $n=4$ seem to be in line with the prediction of this model that the rates will decay slowly with $\mathrm{n}$ in this range of quantum numbers. Moreover, the range of sulfur-to-nitrogen distances $R_{\mathrm{NS}}$ for the model compounds shown in Figures 5 and 6 (i.e., $4-11 \AA$ ) are similar to the $\langle r\rangle$ values for $n=3,4$, and 5 Ry orbitals as shown in Table 1 .

It is also possible to develop an analogous analytical model ${ }^{30}$ governing the rate of through-space electron transfer between pairs of Rydberg orbitals, a process that was shown in our earlier work $^{5 \mathrm{p}, \mathrm{s}}$ to allow Rydberg-attached electrons to migrate from one positive site to another. Again, we model the Rydberg orbitals' radial distribution as in Figure 9 in terms of shells of uniform density located at distances characterized by an average radius and a thickness $\mathrm{T}$ as defined earlier.

Consider, as in Figure 10, two Rydberg s orbitals ${ }^{31}$ having radii $r_{m}>r_{n}$ and thicknesses ${ }^{32} T_{m}>T_{n}$ and with a separation $R$ between their centers. When $R=r_{m}+r_{n}$, the outermost part of each orbital's shell barely touches that of the other, and so there is no overlap between the two orbitals. Within the range of separations $r_{m}+r_{n}-T_{n}>R>r_{m}+r_{n}-T_{n}-T_{m}$ much volume of orbital n's shell will be inside the shell of orbital $m$, and the overlap will be largest when $R=r_{m}+r_{n}-T_{n}-T_{m}$.

The overlap integral $S$ between the two approximate wave functions $\Psi_{n}(\mathbf{r})=\left(1 / V_{n}\right)^{1 / 2}$ and $\Psi_{m}(\mathbf{r})=\left(1 / V_{m}\right)^{1 / 2}$ can be evaluated as

$$
S=\int_{\mathrm{red}} \frac{1}{\sqrt{V_{n}}} \frac{1}{\sqrt{V_{m}}}=\frac{V_{\mathrm{red}}}{\sqrt{V_{n}} \sqrt{V_{m}}}
$$

The volume of the region colored in red in Figure 10 is approximately (for $\left.T_{n}<r_{n}\right) V_{\text {red }}=2 \pi r_{n}^{2}(1-\cos \theta) T_{n}$ and the volumes of the two orbitals' shells are approximately $V_{m}=$ $4 \pi r_{m}{ }^{2} T_{m}$, and $V_{n}=4 \pi r_{n}{ }^{2} T_{n}$, respectively. The angle $\theta$ in Figure 10 can be evaluated as

$$
\cos \theta=\frac{R^{2}+r_{n}^{2}-r_{m}^{2}}{2 R r_{n}}
$$

so

$$
1-\cos \theta=\frac{2 R r_{n}+r_{m}{ }^{2}-r_{n}{ }^{2}-R^{2}}{2 R r_{n}}
$$

This allows $V_{\text {red }}$ to be expressed as

$$
V_{\text {red }}=2 \pi r_{n}^{2} T_{n} \frac{2 R r_{n}+r_{m}{ }^{2}-r_{n}{ }^{2}-R^{2}}{2 R r_{n}}
$$

When evaluated at $R=r_{n}+r_{m}-T_{n}-T_{m}$, the distance at which maximum overlap of the shells is expected, $V_{\text {red }}$ reduces to (ignoring the thickness $T_{n}$ of the smaller Rydberg orbital compared to the radius $r_{m}$ of the larger orbital)

$$
\begin{aligned}
V_{\mathrm{red}} & =\frac{2 \pi r_{n} T_{n}\left(T_{m}+T_{n}\right)}{1+\frac{r_{n}}{r_{m}}} \\
& =\pi\left(\frac{a_{0}}{Z}\right)^{3} \frac{n^{2}(n+1 / 2)^{3 / 2}\left(m(m+1 / 2)^{1 / 2}+n(n+1 / 2)\right)}{1+\frac{n(n+1 / 2)}{m(m+1 / 2)}}
\end{aligned}
$$

where the second identity arises from substituting the expressions for the sizes $\left(r_{n}=\langle r\rangle_{n}\right.$ and $\left.r_{m}=\langle r\rangle_{m}\right)$ and for $T_{n}$ and $T_{m}$ given earlier. Substituting the above expressions for $V_{n}, V_{m}$, and $V_{\text {red }}$ into eq 12 allows the square of the overlap integral to be expressed as

$$
S_{n, m}^{2}=\frac{n \sqrt{n+1 / 2}}{8 m \sqrt{m+1 / 2}} \frac{1}{m+1 / 2}\left[\frac{1+\frac{n \sqrt{n+1 / 2}}{m \sqrt{m+1 / 2}}}{1+\frac{n(n+1)}{m(m+1)}}\right]^{2}
$$

Using the assumption that the electron-transfer rates will scale as $H_{1,2}{ }^{2}$ and that $H_{1,2}$ will be proportional to the overlap $S$, we can approximate the rate of transfer from a Rydberg orbital having quantum number $m$ to another with quantum number $n$ as

$$
\begin{aligned}
\text { rate }_{m \rightarrow n}=\operatorname{rate}_{3 \rightarrow 3} \frac{n \sqrt{n+1 / 2}}{m \sqrt{m+1 / 2}} \frac{3+1 / 2}{m+1 / 2} \times & {\left[\frac{1+\frac{n \sqrt{n+1 / 2}}{m \sqrt{m+1 / 2}}}{1+\frac{n(n+1)}{m(m+1)}}\right]^{2} }
\end{aligned}
$$

This result suggests that electron-transfer rates among excited Rydberg orbitals will be highest for $n=m=3$ and will decay as $n$ or $m$ increases. More specifically, it suggests that

(a) transfer rates between Rydberg orbitals having the same quantum number $(n=m>3)$ should decrease with $m$ as rate r $_{m \rightarrow m}$ $\propto(3+1 / 2) /(m+1 / 2)$ and

(b) transfer rates between Rydberg orbitals with (fixed) quantum number $n$ and those having a different quantum number $m$ should decrease as $m$ increases as rate $_{m \rightarrow n} \propto\left[n(n+1 / 2)^{1 / 2}\right] /$ $\left[m(m+1 / 2)^{3 / 2}\right]$.

So, the fastest transfer rates $^{33}$ are expected for transfer between pairs of Rydberg orbitals having the same quantum numbers, but these rates should decrease slowly (as rate m $\rightarrow m \propto$ $(3+1 / 2) /(m+1 / 2)=0.78,0.64,0.33$, and 0.17 for $m=4,5$, 10 , and 20 , respectively) as that quantum number grows. These observations suggest that one should focus on transitions between Rydberg orbitals (i) having equal quantum numbers 
and (ii) having just enough radial extent to span the distance $R$ between their respective centers and to generate optimal overlap (i.e., $R \approx 2 r_{n}-2 T_{n}$ tells which $n$ value will be optimal for a given $R$ ).

D. Overview of Conclusions Regarding ETD/ECD Mechanisms. We believe the analysis just offered makes a very important contribution to our view of intrapeptide electron transfer. It suggests the following (see Figure 6):

a. As long as an SS $\sigma^{*}$ orbital experiences sufficient Coulomb stabilization (ca. $1 \mathrm{eV}$ ) to cause the $\sigma^{*}$-attached state's curve to lie below the parent's curve at the parent's equilibrium geometry (e.g., see Figure 6), intrapeptide electron transfer to that $\mathrm{SS} \sigma^{*}$ orbital is possible. An analogous statement also holds for OCN $\pi^{*}$ orbitals, although these orbitals require more Coulomb stabilization (ca. $25 \mathrm{eV}$ ) than do SS $\sigma^{*}$ orbitals.

b. In this case, regardless (within $3<n<20$ ) of what range of Rydberg states the SS $\sigma^{*_{-}}$(or amide $\pi^{*_{-}}$) attached state crosses near the equilibrium geometry of the parent ion, there will be at least one such Rydberg state with sufficient $H_{1,2}$ coupling strength to facilitate intrapeptide electron transfer at a rate considerably in excess of $10^{6} \mathrm{~s}^{-1}$.

c. The particular Rydberg orbitals that will dominate in such intrapeptide electron transfer will be those whose radial extent $\langle r\rangle=n(n+1 / 2) a_{0} / Z$ is close to the distance $R_{\mathrm{NS}}$ separating the Rydberg orbital and the SS (or OCN) bond site. For example, even though Rydberg orbitals with $n=20$ are capable of inducing electron transfer over ca. $200 \AA$, if an SS bond is within $15 \AA$ of a positive site, it will be (see Table 1 ) the $n=4,5$, and 6 Ry orbitals on that positive site that dominate because they have their maximum radial density in this distance range.

d. Electron transfer from a Rydberg orbital on one positive site to a Rydberg orbital on another positive site is possible, as we showed earlier. ${ }^{5 \mathrm{p}, \mathrm{s}}$ These rates are expected to be largest when the principal quantum numbers of the two Rydberg orbitals are the same and to decrease (but only slowly as rate $_{m \rightarrow m} \propto(3$ $+1 / 2) /(m+1 / 2))$ as the principal quantum numbers of the Rydberg orbitals grow. The Rydberg orbital most efficient in facilitating inter-Rydberg electron transfer will be that having just enough radial extent to span the distance $R$ between their respective centers and to generate optimal overlap (i.e., that Rydberg orbital whose $\mathrm{n}$ satisfies $R \approx 2 r_{n}-2 T_{n}$ ).

The view outlined above thus strongly suggests that intrapeptide electron transfer from Rydberg to SS (or OCN, we anticipate) sites can occur over very long distances because even $n=8$ or 20 Ry orbitals, which have $\langle r\rangle=36 \AA$ or $217 \AA$, respectively, can have strong enough $H_{1,2}$ coupling to induce electron transfer well within $10^{-6} \mathrm{~s}$. However, it is more probable that Rydberg orbitals having smaller $n$ values will dominate because any SS $\sigma^{*}$ or amide $\pi^{*}$ orbital that is susceptible to electron attachment likely has one or more positive sites closer than $36-217 \AA$; a single positive site this far away could not generate sufficient Coulomb stabilization to make the SS $\sigma^{*}$ or amide $\pi^{*}$ orbital capable of binding an electron. Most probably, it will be Rydberg orbitals with radial extents near $14 \AA$ (for SS $\sigma^{*}$ attachment) or near $6.5 \AA$ (for amide $\pi^{*}$ attachment) and thus $n=4,5$, or 6 that will play dominant roles. Even in the somewhat unusual case (where two + charges each ca. $30 \AA$ away generate the needed Coulomb stabilization) presented by the $\left(\mathrm{AcCA}_{20} \mathrm{~K}+\mathrm{M}\right)_{2}{ }^{2+}$ compounds shown in Figure 4 , Table 1 suggests it is probably the $n=7$ or 8 Ry orbitals that have proper radial extent to span this gap.

\section{Summary}

The body of work encompassed by our earlier efforts, combined with the findings reported here, suggests that the following steps are important in ETD and ECD fragmentation of positively charged polypeptides:

1. Initial electron attachment ${ }^{5 \mathrm{~h}, \mathrm{i}, \mathrm{m}}$ most likely (90-99\%) takes place into a Rydberg orbital located on one ${ }^{9}$ of the peptide's positive sites, although a small $(1-10 \%)$ fraction of the attachment events (and subsequent SS or $\mathrm{N}-\mathrm{C}_{\alpha}$ bond cleavage) can occur into SS $\sigma^{*}$ or amide $\pi^{*}$ orbitals that have sufficient (ca. $1 \mathrm{eV}$ for $\mathrm{SS}$ and $2.5 \mathrm{eV}$ for amide) Coulomb stabilization.

2. Occupation of a ground $3 \mathrm{~s}$ Rydberg orbital on a protonated amine site will result in prompt (ca. $10^{-9} \mathrm{~s}$ ) H-atom loss $\left(\mathrm{R}-\mathrm{NH}_{3} \rightarrow \mathrm{RNH}_{2}+\mathrm{H}\right)$ or $\mathrm{NH}_{3}$ loss and thus to termination of the possibility of $\mathrm{SS}$ or $\mathrm{N}-\mathrm{C}_{\alpha}$ bond cleavage.

3. Attachment into any excited Rydberg orbital is followed by

a. a cascade of radiationless transition events to lower-energy Rydberg levels on time scales of ca. $10^{-6} \mathrm{~s}$ per transition, ${ }^{34}$ during which

b. electron transfer from the excited Rydberg orbital to an SS $\sigma^{*}$ or amide $\pi^{*}$ orbital can take place at rates exceeding $10^{6} \mathrm{~s}^{-1}$ (and after which $\mathrm{SS}$ or $\mathrm{N}-\mathrm{C}_{\alpha}$ bond cleavage occurs) even for Rydberg orbitals having principal quantum numbers as high as 20 . However, only SS $\sigma^{*}$ or amide $\pi^{*}$ orbitals that experience Coulomb stabilization exceeding 1 or $2.5 \mathrm{eV}$, respectively, can act as electron acceptors in such transfers.

4. The only excited Rydberg orbitals that can cause such electron-transfer events are those that have

a. $n<$ ca. 20 (so that the electron-transfer rate exceeds $10^{6}$ $\left.\mathrm{s}^{-1}\right)$

b. radial size $\langle r\rangle=n(n+1 / 2) a_{0} / Z$ close to their distance to the SS or amide bond site so that Rydberg-valence orbital overlap is favorable, and

c. vertical (i.e., near the equilibrium geometry of the parent ion) energies very similar to the vertical energy of the SS $\sigma^{*}$ or amide $\pi^{*}$-attached state.

5. It is also possible for an electron initially attached into an excited Rydberg orbital on one positive site to undergo transfer ${ }^{5 p^{-s}}$ to a Rydberg orbital (having similar or lower energy) on a nearby positive site. Such processes allow attached electrons to migrate throughout the polypeptide. These rates are expected to be largest when the principal quantum numbers of the two Rydberg orbitals are similar and to decrease slowly as the principal quantum numbers of the Rydberg orbitals grow.

The "big picture" outlined above is consistent with the observation that disulfide and $\mathrm{N}-\mathrm{C}_{\alpha}$ bond cleavage is found to occur throughout much of the peptide backbone under ECD or ETD conditions because it suggests that even Rydberg orbitals with $n$ as high as 8-20 can facilitate electron transfer (over distances as large as ca. 36-217 $\AA$, respectively) as long as the corresponding SS and OCN sites experience Coulomb stabilizations in excess of 1 and $2.5 \mathrm{eV}$, respectively. In closing this Summary, we wish to offer suggestions as to experiments that could be performed to gain further testing of the ideas offered here.

Specifically, we suggest it would be helpful to, for example, carry out further experiments on $\left(\mathrm{AcCA}_{k} \mathrm{~K}+\mathrm{M}\right)_{2}{ }^{2+}$ model systems similar to those shown in Figure 4, because such compounds possess substantial geometrical rigidity (so distances among various sites do not fluctuate much), contain both SS and $\mathrm{N}-\mathrm{C}_{\alpha}$ bonds, and have two (potentially distinct) charged sites. For example:

1. We envision ETD experiments using electron donors having different electron binding strengths (but similar steric bulk so steric factors do not become an issue) to limit the principal quantum number range of the Rydberg orbitals 
involved in the electron transfer. For example, ETD donors having binding energies in excess of $1 \mathrm{eV}$ could not populate $n>5$ Ry states (having $Z=1$ ). Rydberg orbitals with $n=5$ have $\langle r\rangle=15 \AA$, so electron transfer should be limited to approximately this distance and thus SS cleavage should not be observed in $\left(\mathrm{AcCA}_{k} \mathrm{~K}+\mathrm{M}\right)_{2}{ }^{2+}$ when $k>10$ (because then the distance from Lys to the SS bond exceeds $18 \AA$ ) when such an ETD donor is employed. In contrast, using an ETD donor having a lower electron binding energy can populate higher- $n$ Rydberg states, thus allowing transfer to an SS bond that is more distant (e.g., Table 1 suggests that an $n=7$ Ry orbital can span the ca. $30 \AA$ separating the Lys and SS sites in $\left.\left(\mathrm{AcCA}_{20} \mathrm{~K}+\mathrm{M}\right)_{2}{ }^{2+}\right)$.

2. It would be good if the terminal Lys amino acids were replaced by an artificial amino acid having a shorter basic side chain (e.g., $-\mathrm{CH}_{2}-\mathrm{NH}_{2}$; or as in ref 12 where protonated disulfide-linked dimers of Ac-Cys-Ala $k_{k}-\mathrm{NH}_{2}$ were used) so the positively charged sites' locations would undergo smaller thermal displacements, and thus could be more precisely specified.

3. Asymmetric species such as disulfide-linked [(M+KA $\mathrm{AAc}_{k} \mathrm{CA}$ $\left.\left(\mathrm{AcCA}_{l} \mathrm{~K}+\mathrm{M}^{\prime}\right)\right]^{q+}$ could be used, and the lengths $(k$ and $l)$ of the Ala helices as well as the nature of the charges $\mathrm{M}$ and $\mathrm{M}^{\prime}$ could be varied. We note that, for $k=10,15$, and 20, the distances ${ }^{12}$ from the terminal Lys nitrogen atoms to the SS bond of the species shown in Figure 4 were estimated to be ca. 18, 24, and $32 \AA$, respectively; all provide sufficient Coulomb stabilization at the SS site and at the four OCN sites closest to the Lys termini (i.e., within ca. $6.5 \AA$ ) to permit $\mathrm{SS}$ or $\mathrm{N}-\mathrm{C}_{\alpha}$ bond cleavage, as was observed at these locations and reported in ref 12 .

4. The charges on the $M$ and $M^{\prime}$ species in disulfide-linked $\left[\left(\mathrm{M}+\mathrm{KA}_{k} \mathrm{CAc}\right)\left(\mathrm{AcCA}_{l} \mathrm{~K}+\mathrm{M}^{\prime}\right)\right]^{q+}$ could be varied (e.g., with $\mathrm{M}$ being $\mathrm{H}^{+}$but $\mathrm{M}^{\prime}$ being $\mathrm{Ca}^{2+}$ ). Our model would predict $\mathrm{N}-\mathrm{C}_{\alpha}$ cleavage further (i.e., within $13 \AA$ ) from the Lys terminus of $\left(\mathrm{AcCA}_{l} \mathrm{~K}+\mathrm{M}^{\prime}\right)$ than in $\left(\mathrm{M}+\mathrm{KA}_{k} \mathrm{CAc}\right)$ but with SS cleavage in all cases with $\mathrm{k}$ and $l<20$. It would also predict that $\mathrm{SS}$ cleavage should occur for disulfide-linked $\left[\left(\mathrm{KA}_{k} \mathrm{CAc}\right)\left(\mathrm{AcCA}_{l} \mathrm{~K}+\mathrm{Ca}\right)\right]^{2+}$ for $l<20$ and that $\mathrm{N}-\mathrm{C}_{\alpha}$ cleavage should occur within ca. 13 $\AA$ of the $\mathrm{Ca}^{2+}$ site but that $\mathrm{N}-\mathrm{C}_{\alpha}$ cleavage should not occur within the $\left(\mathrm{KA}_{k} \mathrm{CAc}\right)$ portion of this molecule.

5. It would be interesting to carry out ETD experiments (again using anion donors of varying electron binding strengths) on polypeptides of a fixed sequence structure but having a range of charge states. One expects ${ }^{35}$ that, for parent ions in low charge states, a smaller fraction of OCN $\pi^{*}$ orbitals will be sufficiently Coulomb stabilized to undergo electron attachment. Higher charge states should render more OCN $\pi^{*}$ orbitals active thus generating a higher degree of $\mathrm{N}-\mathrm{C}_{\alpha}$ cleavage.

It is our hope that experimental groups will be sufficiently inspired by the specific predictions that our model provides to undertake tests similar to (or better than) those we suggest above.

Acknowledgment. This work has been supported by NSF Grant No. 0806160. Significant computer time provided by the Center for High Performance Computing at the University of Utah is also gratefully acknowledged.

\section{References and Notes}

(1) In Simons, J.; Smith, W. D. J. Chem. Phys. 1973, 58, 4899-4907, the equations of motion (EOM) method was developed.

(2) Simons, J. J. Phys. Chem. A 2008, 112, 6401-6511.

(3) (a) Zubarev, R. A.; Kelleher, N. L.; McLafferty, F. W. J. Am. Chem. Soc. 1998, 120, 3265-3266. (b) Zubarev, R. A.; Kruger, N. A.; Fridriksson, E. K.; Lewis, M. A.; Horn, D. M.; Carpenter, B. K.; McLafferty, F. W. J. Am. Chem. Soc. 1999, 121, 2857-2862. (c) Zubarev, R. A.; Horn, D. M.;
Fridriksson, E. K.; Kelleher, N. L.; Kruger, N. A.; Lewis, M. A.,; Carpenter, B. K.; McLafferty, F. W. Anal. Chem. 2000, 72, 563-573. (d) Zubarev, R. A.; Haselmann, K. F.; Budnik, B.; Kjeldsen, F.; Jensen, R. Eur. J. Mass Spectrom. 2002, 8, 337-349.

(4) (a) Syka, J. E. P.; Coon, J. J.; Schroeder, M. J.; Shabanowitz, J.; Hunt, D. F. Proc. Natl. Acad. Sci. 2004, 101, 9528-9523. (b) Coon, J. J.; Syka, J. E. P.; Schwartz, J. C.; Shabanowitz, J.; Hunt, D. F. Int. J. Mass Spectrom. 2004, 236, 33-42. (c) Pitteri, S. J.; Chrisman, P. A.; McLuckey, S. A. Anal. Chem. 2005, 77, 5662-5669. (d) Gunawardena, H. P.; He, M.; Chrisman, P. A.; Pitteri, S. J.; Hogan, J. M.; Hodges, B. D. M.; McLuckey, S. A. J. Am. Chem. Soc. 2005, 127, 12627-12639. (e) Gunawardena, H. P.; Gorenstein, L.; Erickson, D. E.; Xia, Y.; McLuckey, S. A. Int. J. Mass. Spectrom. 2007, 265, 130-138.

(5) (a) Syrstad, E. A.; Turecek, F. J. Phys. Chem. A 2001, 105, 1114411155. (b) Turecek, F.; Syrstad, E. A. J. Am. Chem. Soc. 2003, 125, 33533369. (c) Turecek, F.; Polasek, M.; Frank, A.; Sadilek, M. J. Am. Chem. Soc. 2000, 122, 2361-2370. (d) Syrstad, E. A.; Stephens, D. D.; Turecek, F. J. Phys. Chem. A 2003, 107, 115-126. (e) Turecek, F. J. Am. Chem. Soc. 2003, 125, 5954-5963. (f) Syrstad, E. A.; Turecek, F. Am. Soc. Mass. Spectrom. 2005, 16, 208-224. (g) Uggerud, E. Int. J. Mass. Spectrom. 2004, 234, 45-50. (h) Anusiewicz, I.; Berdys-Kochanska, J.; Simons, J. J. Phys. Chem. A 2005, 109, 5801-5813. (i) Anusiewicz, I.; Berdys-Kochanska, J.; Skurski, P.; Simons, J. J. Phys. Chem. 2006, A110, 1261-1266. (j) Sawicka, A.; Skurski, P.; Hudgins, R. R.; Simons, J. J. Phys. Chem. B 2003, 107, 13505-13511. (k) Sobczyk, M.; Skurski, P.; Simons, J. Adv. Quantum Chem. 2005, 48, 239-251. (1) Sawicka, A.; Berdys-Kochaska, J.; Skurski, P.; Simons, J. Int. J. Quantum Chem. 2005, 102, 838-846. (m) Anusiewicz, I.; Berdys, J.; Sobczyk, M.; Sawicka, A.; Skurski, P.; Simons, J. J. Phys. Chem. A 2005, 109, 250-258. (n) Bakken, V.; Helgaker, T.; Uggerud, E. Eur. J. Mass Spectrom. 2004, 10, 625-638. (o) Skurski, P.; Sobczyk, M.; Jakowski, J.; Simons, J. Int. J. Mass. Spectrom 2007, 265, 197-212. (p) Sobczyk, M.; Neff, D.; Simons, J. Int. J. Mass Spectrom. 2008, 269, 149164. (q) Sobczyk, M.; Simons, J. Int. J. Mass. Spectrom. 2006, 253, $274-$ 280. (r) Sobczyk, M.; Simons, J. J. Phys. Chem. B 2006, 110, 7519-7527. (s) Neff, D.; Sobczyk, M.; Simons, J. Int. J. Mass Spectrom. 2008, 276, 91-101. (t) Neff, D.; Simons, J. Int. J. Mass Spectrom. 2008, 277, 166174. (u) Turecek, F.; Chen, X.; Hao, C. J. Am. Chem. Soc. 2008, 130, 88188833. (v) Chen, X.; Turecek, F. J. Am. Chem. Soc. 2006, 128, 12520 12530. (w) Holm, A. I. S.; Larsen, M. K.; Panja, S.; Hvelplund, P.; Brøndsted Nielsen, S.; Leib, R. D.; Donald, W. A.; Williams, E. R.; Hao, C.; Tureèek, F. Int. J. Mass Spectrom. 2008, 276, 116-126. (x) ChamotRooke, J.; Malosse, C.; Frison, G.; Tureèek, F. J. Am. Assoc. Mass Spectrom. 2007, 18, 2146-2161. (y) Fung, Y.M. E.; Chan, T.-W. D. J. Am. Assoc. Mass Spectrom. 2005, 16, 1523-1535. (z) Konishi, H.; Yokotake, Y.; Ishibahsia, T. J. Mass Spectrom. Soc. Jpn. 2002, 50, 229-232. (za) Holm, A. I. S.; Hvelplund, P.; Kadhane, U.; Larsen, M. K.; Liu, B.; Nielsen, S. B.; Panja, S.; Pedersen, J. M.; Skryudstrup, T.; Støchkel, K.; Williams, E. R.; Worm, E. S. J. Phys. Chem. A 2007, 111, 9641-9643.

(6) Actually, it is the energies of the electron-attached states in which an electron occupies the SS $\sigma^{*}$ or amide $\pi^{*}$ orbital that are lowered.

(7) Frison, G.; van der Rest, G.; Turecek, F.; Besson, T.; Lemaire, J.; Matre, P.; Chamot-Rooke, J. J. Am. Chem. Soc. 2008, 130, 1491614917.

(8) Dezarnaud-Dandine, C.; Bournel, F.; Troncy, M.; Jones, D.; Modelli, A. J. Phys. B: At. Mol. Opt. Phys. 1998, 31, L497-L502. Seydou, M.; Modelli, A.; Lucas, B.; Konate, K.; Desfrancois, C.; Schermann, J. P. Eur. Phys. J. D 2005, 35, 199-205.

(9) In ref $5 u$, it is shown that electron attachment to species with more than one positively charged sites might generate Rydberg states consisting of superpositions of orbitals localized on two or more of the charged centers. See, for example, the $\mathrm{X}$ and $\mathrm{A}$ state-orbitls shown in Figure 4 of ref $5 \mathrm{u}$. Because such superposition states are close in energy (i.e., for the case with two positive centers, they consist of constructive and destructive combinations of Rydberg orbitals located on each of the two centers and differ by only $0.17 \mathrm{eV}$ for the case shown in Figure 4 of ref $5 \mathrm{u}$ ), an ETD or ECD initial electron attachment event can be expected to populate either or both of these states.

(10) These $H_{1,2}$ coupling strengths are obtained by carrying out a series of calculations at much finer $R$ value separations than shown in Figure 3 and searching for the smallest energy splitting $(\Delta)$ between the two adiabatic energies. $H_{1,2}$ is then taken as $\Delta / 2$.

(11) The SS bond vibration occurs near $600 \mathrm{~cm}^{-1}$, so $v \approx 600 \times 3 \times$ $1010=1.8 \times 1013 \mathrm{~s}^{-1}$

(12) Hudgins, R.; Håkansson, K.; Quinn, J. P.; Hendrickson, C. L.; Marshall, A. G. In Proceedings of the 50th ASMS Conference on Mass Spectrometry and Allied Topics, Orlando, Florida, 2002. A020420. Figure 1 first appears in publication in ref $5 \mathrm{j}$.

(13) To illustrate how we arrive at this conclusion, we note it was shown in ref $5 \mathrm{q}$ that coupling strengths in the $300 \mathrm{~cm}^{-1}$ range produce surfacehopping probabilities (using Landau-Zener theory) of ca. $0.1-0.5$. Thus, we can estimate the rates of electron transfer by multiplying the $S-S$ vibrational frequency $v_{\mathrm{SS}}\left(\mathrm{ca} .1 .5 \times 10^{13} \mathrm{~s}^{-1}\right)$ by the surface hopping 
probability $(0.1-0.5)$ and then scaling by the ratio of the square of $\left(H_{1.2} / 300\right)$ :

(a)

$$
\text { rate } \approx(1.5-7.5) \times 10^{12}\left(H_{1.2} / 300\right)^{2} \mathrm{~s}^{-1}
$$

For the through-bond migration to be effective in cleaving the $\mathrm{S}-\mathrm{S}$ bond, it must occur before the Rydberg species from which the electron is transferred can decay by some other mechanism. It is believed that electron attachment (in ECD or ETD) at a positively charged side chain initially occurs into an excited Rydberg orbital after which a decay cascade leads to formation of the ground Rydberg species. It is known that excited Rydberg states belonging to protonated or fixed-charge amine site undergo radiationless relaxation to the ground Rydberg state in a few to several milliseconds. Moreover, we know that the excited Rydberg states do not decay by $\mathrm{N}-\mathrm{H}$ or $\mathrm{N}-\mathrm{C}_{\mathrm{a}}$ bond cleavage, but the ground Rydberg states do (in ca. $10^{-9} \mathrm{~s}$ ). Hence, to be effective in cleaving an $\mathrm{S}-\mathrm{S}$ bond, the throughbond electron transfer must occur within ca. $10^{-6} \mathrm{~s}$ of the time the electron attaches to an excited Rydberg orbital. This fact allows us to estimate the smallest $H_{1,2}$ coupling strength that could produce $\mathrm{S}-\mathrm{S}$ bond cleavage by solving eq a for a rate of ca. $10^{6} \mathrm{~s}^{-1}$. This gives an estimate of $H_{1,2}$ min $=$ $0.11-0.24 \mathrm{~cm}^{-1}$. Using our earlier $\mathrm{H}_{1.2}$ data, which we emphasize pertain only to $3 \mathrm{~s}$ or $3 \mathrm{p}$ Rydberg orbitals, we concluded that through-bond electron transfer can occur at a rate capable of yielding $\mathrm{S}-\mathrm{S}$ bond cleavage if there are up to 7 bonds (aliphatic or olefinic) or ca. $15 \AA$.

(14) The ground $3 \mathrm{~s}$ Rydberg state of $\mathrm{R}-\mathrm{NH}_{3}$ undergoes bond cleavage because this state's electronic configuration (which can be denoted $\sigma_{\mathrm{N}-\mathrm{H}^{2}}$ $3 \mathrm{~s}^{1}$, meaning having one electron in a Rydberg orbital of $3 \mathrm{~s}$ character and two electrons in an $\mathrm{N}-\mathrm{H} \sigma$ bonding orbital) correlates directly with the $\mathrm{H}$ $+\mathrm{R}-\mathrm{NH}_{2}$ with on electron in an $\mathrm{H}$ atom $1 \mathrm{~s}$ orbital and two electrons in the $\mathrm{R}-\mathrm{NH}_{2}$ nitrogen lone pair orbital. The excited Rydberg states do not correlate with these ground-state products and thus have large barriers to bond cleavage.

(15) Holm, A. I. S.; Hvelplund, P.; Kadhane, U.; Larsen, M. K.; Liu, B.; Nielsen, S. B.; Panja, S.; Pedersen, J. M.; Skrydstrup, T.; Støchkel, K.; Williams, E. R.; Worm, E. S. J. Phys. Chem. A 2007, 111, 9641-9643.

(16) Neff, D.; Smuczynska, S.; Simons, J. Int. J. Mass Spectrom. 2009, $283,122-134$

(17) Thus far in our studies, we have limited consideration to positive sites involving protonated amines. Clearly, when the charged site involves a His or Arg side chain, the strength of the binding of the attached electron will be different and thus one can expect different rates of electron transfer from such sites to SS or OCN sites. We plan to investigate the analogous intramolecular electron-transfer events involving such side chains in future work.

(18) (a) Gutowski, M.; Simons, J. J. Chem. Phys. 1990, 93, 3874-3880. (b) Skurski, P.; Gutowski, M.; Simons, J. Int. J. Quantum Chem. 2000, 80, 1024-1038. 6806

(19) Kendall, R. A., Jr.; Harrison, R. J. J. Chem. Phys. 1992, 96, 6796-

(20) Frisch, M. J.; Trucks; G. W.; Schlegel, H. B.; Scuseria, G. E.; Robb, M. A.; Cheeseman, J. R.; Montgomery, J. A., Jr.; Vreven, T.; Kudin, K. N.; Burant, J. C.; Millam, J. M.; Iyengar, S. S.; Tomasi, J.; Barone, V.; Mennucci, B.,;Cossi, M.; Scalmani, G.; Rega, N.; Petersson, G. A.; Nakatsuji, H.; Hada, M.; Ehara, M.; Toyota, K.; Fukuda, R.; Hasegawa, J. Ishida, M.; Nakajima, T.; Honda, Y.; Kitao, O.; Nakai, H.; Klene, M.; Li, X.; Knox, J. E.; Hratchian, H. P.; Cross, J. B.; Adamo, C.; Jaramillo, J.; Gomperts, R.; Stratmann, R. E.; Yazyev, O.; Austin, A. J.; Cammi, R.; Pomelli, C.; Ochterski, J. W.; Ayala, P. Y.; Morokuma, K.; Voth, G. A.; Salvador, P.; Dannenberg, J. J.; Zakrzewski, V. G.; Dapprich, S.; Daniels, A. D.; Strain, M. C.; Farkas, O.; Malick, D. K.; Rabuck, A. D.; Raghavachari, K.; Foresman, J. B.; Ortiz, J. V.; Cui, Q.; Baboul, A. G.; Clifford, S.; Cioslowski, J.; Stefanov, B.; Liu, B.; Liashenko, A.; Piskorz, P.; Komaromi, I.; Martin, R. L.; Fox, D. J.; Keith, T.; Al-Laham, M. A.; Peng, C. Y.; Nanayakkara, A.; Challacombe, M.; Gill, P. M. W.; Johnson, B.; Chen, W.; Wong, M. W.; Gonzalez, C.; Pople, J. A. Gaussian, v. 03; Gaussian, Inc.: Wallingford CT, 2004.

(21) Schaftenaar, G.; Noordik, J. H. J. Comput. Aided Mol. Des. 2000 , 14,123

(22) In ref $5 \mathrm{~s}$ we studied electron transfer in model systems $\mathrm{Me}-\mathrm{SS}-(\mathrm{HC}=\mathrm{CH})-\left(\mathrm{CH}_{2}\right)_{n}-\mathrm{NH}_{3}{ }^{+}$containing one $\mathrm{CC}$ double bond. However, because these compounds also contained methylene spacer units, delocalization of the negative charge from the SS $\sigma^{*}$-attached state was limited. As a result, the high degree of delocalization observed in the present paper was not observed.

(23) As discussed later, Rydberg orbitals have their highest radial electron density at distances far from their centroid.

(24) To be more precise, the quantum number shown as $n$ is usually replaced by an effective principle quantum number $(n-\delta)$ when describing Rydberg orbitals, where the quantum defect $\delta$ is supposed to account for the screening and penetration effects from inner-shell electrons. In this paper, we will retain the hydrogenic notation, using $n$ as the quantum number, for simplicity. In addition, we should note that the radial behavior of Rydberg orbitals shown in eq 5 is correct only for large $r$; at smaller distances, the radial wave function oscillates in sign to retain its orthogonality to lower- $n$ wave functions. However, the amplitudes of the peaks in the wave function decrease as one moves inward from its outermost peak.

(25) The average radial extent $\langle r\rangle=n(n+1 / 2) a_{0} / Z$ and the location $r_{\max }=n 2 a_{0} / Z$ of the maximum in the radial distribution differ little, so we do not distinguish between them in this analysis.

(26) To be more precise, we should not use the term "overlap" because this expression would allow for cancellation of positive and negative contributions. Because we are attempting to develop an approximation to the $H_{1,2}$ couplings between a Rydberg orbital and a valence (SS $\sigma^{*}$ or OCN $\pi^{*}$ ) orbital that is subsumed within the Rydberg orbital, we should say we wish to calculate the amount of three-dimensional space shared by the two orbitals.

(27) In addition to using a simplified description of the Rydberg orbitals' radial probability distribution and ignoring angular "shape" dependence, there are two other significant assumptions underlying this expression for the overlap. First, we assumed that the distance R separating the Rydberg orbital's centroid and that of the valence (SS $\sigma^{*}$ or OCN $\pi^{*}$ ) orbital is such that the valence orbital resides essentially entirely within the region where the Rydberg orbital has its major amplitude. Second, we assumed that the size of the valence orbital (as characterized by the parameter $x$ ) does not vary significantly with this inter-atomic distance. Because the electron binding energy (EA) of the SS $\sigma^{*}$-attached (or OCN $\pi^{*}$-attached) state does depend on the distances to nearby positively charged groups, the size of these orbitals will depend on $R$; however, because the radial extent of an orbital is known to depend on its electron binding energy EA as exp $\left(-(2 E A)^{1 / 2} r\right)$, this variation is expected to be rather weak over the limited range of distances within which the first assumption applies.

(28) Our strategy is to (i) use knowledge of the $H_{1,2}$ values corresponding to the strong-overlap situation (e.g., as shown in the green figures in Figure 8) to establish the maximum rates appropriate to the $n=3$ Ry orbitals (because it is for such orbitals that we have been most successful in calculating $H_{1,2}$ in an ab initio manner), (ii) estimate the maximum rates appropriate to $n>3$ Ry orbitals using our analytical scaling law (eq 11), and (iii) realize that each of these maximum rates will decay exponentially with distance as the valence orbital moves beyond the region where the Rydberg orbital has most of its amplitude (between $\langle r\rangle-T / 2$ and $\langle r\rangle+$ $T / 2)$.

(29) We use the phrase maximum to denote the rates which result when the valence orbital is entirely subsumed by the Rydberg orbital.

(30) In this case, where we have approximate analytical expressions for both Rydberg orbitals, it is possible to obtain an analytical expression for the overlap integral as a function of the distance $R$ between the two orbitals and their principal quantum numbers. However, the result is rather cumbersome, and we prefer to retain contact with the intuitive picture of Rydberg orbitals having shells of thickness $T$ and radial extent $\langle r\rangle$ that we used to treat the Rydberg-valence overlap case.

(31) The treatment of overlaps between pairs of Rydberg orbitals of arbitrary angular momentum quantum numbers $(l, m)$ would be more cumbersome. However, the main difference between the more general cases and the s-orbital case treated here will be to introduce factors depending on $l$ and $m$ that will not tend to change the gross features (i.e., the dependence on the $n$ quantum numbers of the two orbitals) we seek to uncover here.

(32) In this derivation, we view each Rydberg orbital as having a shell of thickness $T$ that extends radially inward from $\langle r\rangle$ to $\langle r\rangle-T$. This is slightly different from how we treated the Rydberg orbitals in deriving the Rydbergvalence overlap integral where we used a shell ranging from $\langle r\rangle-T / 2$ to $\langle t\rangle+T / 2$, but because $T<\langle r\rangle$, these differences should not alter the qualitative conclusions (i.e., how rates vary with $n$ and $m$ ) that we are seeking.

(33) In the case of Rydberg-to-valence (i.e., SS $\sigma^{*}$ or $\mathrm{OCN} \pi^{*}$ ) electron transfer treated earlier, we were able to estimate the absolute rates (i.e., using eq 11) using LZ theory in terms of the probability $P$ of transfer multiplied by the frequency $n$ at which a surface crossing occurs. The surface crossing frequency was taken to be the frequency of vibration of the SS bond for SS $\sigma^{*}$ attachment. However, for Rydberg-to-Rydberg transfers, we are limited by the fact that it is difficult to estimate the frequencies at which a pair of Rydberg orbitals on different charged sites approach and overlap one another. Therefore, in this analysis, we focus mainly on determining which Rydberg orbitals should be most effective in facilitating electron transfer for a given distance $R$ between the two charged sites and on how the rates of transfer between such sites will depend on the $n$ quantum numbers of the two Rydberg orbitals.

(34) See ref $5 f$ as well as: Turecek, F.; Reid, P. J. Int. J. Mass Spectrom. 2003, 222, 49-61.

(35) This assumes that the charges are spatially distributed in a way that minimizes the internal Coulomb repulsions among positive sites.

JP9057059 